\title{
Perturbazioni geomagnetiche, aurorali, ionosferiche e dei raggi cosmici : interdipendenze e relazioni con l'attività solare
}

\author{
F. Mariasi $(*)-$ F. Molixa $(* *)$
}

Ricevuto il 30 Aprile 1966

\begin{abstract}
Riassunto. - ln relazione all esistenza, accertata sperinentahmente (Cap. V a), di canpi magnetie interplanetari cliversi dal campo geomagnetico e alla ipotesi delle nuvole di phasma come causa di essi, si prendono in esame i eampi magnetici soluri, in vista della possibiliti di una loro estensione nello spazio. Siccone con i campi solari pir iutensi sono associati i fenomeni che danno luogo a effetti terrestri, si descrivono, fra tali fenomeni, gli eventi di radioentissione, the lammo strette relazioni sia con i snddetti campi magnetici sia con l'enissione di plasmat.

Prima di discutere $\mathrm{i}$ dati sperimentali, diretti e indiretti. relativi alle nuvole di plasma enesse dal sole. si passano in rassegna gli eventi connessi con l'arrivo noll'amosfera terestre di protoni solari di 10 -100 $\mathrm{MeV}$, i cosidetti PCA (P'olar Cap Absorption), di ani si esanina la morfologia, la distribuzione geografica e le relazioni con grli eventi solari. si descrivono infine i risultati finora ottenuti delle misure dirette dei protoni solari effettuate sia a mezzo tli palloni sia a mezzo sli satelliti.
\end{abstract}

Summar. - Existence of interplanetary magnetic fields other than the geomagnetie field is now well establislied. They travel as frozen-in fields insike the solar plasna clourls: matrnetic fields on the sun are then disenssed. Furtherly radioemission phenomena are considered from the viewpoint of their association and correlation with the interplanetary magnetic field and solar plasma phenomena.

Morphology and geographical distribution of Polar Cap Absorption events and their correlations with solar phenomena are reviewed.

At last experimental results on solar protons events are disenssed.

(*) Istituto di Fisien dell' Università di Roma.

(**) Istituto Nazionale di Geofisica, Roma. 


\section{STATO FISICO DELLO SPAZIO INTERPLANETARIO}

\section{b. - CAMPI MAGNETICI E RADIOEMISSIONE SOLARI PROTONI SOLARI}

\subsection{Campi magnetici solari.}

Nei paragrafi precedenti abbiamo studiato i campi magnetici esistenti nello spazio extraterrestre, quali si sono potuti determinare o con inferenze indirette basate soprattutto sul comportamento dei raggi cosmici nelle vicinanze del nostro pianeta, o con misure dirette eseguite mediante satelliti artificiali e sonde spaziali. Abbiamo così visto che non esiste solo il campo magnetico terrestre tendente ad annullarsi a grandi distanze, ma che lo spazio interplanetario appare permeato di campi magnetici pinttosto irregolari e disordinati nei quali il campo magnetico terrestre è immerso.

Ci proponiamo ora di studiare eventuali campi magnetici solari per potere in seguito discutere la possibilità di una loro estensione in modo più o meno ordinato nello spazio interplanetario. Lo studio dei campi magnetici sul Sole è più facile di quello dei ampi interplanetari, e di fatto si è iniziato ver'so il principio di questo secolo, utilizzando l'effetto Zeeman.

5.5.1. Principio della misura. - Schematicamente, il principio dellat misura dei campi magnetici solari è il seguente. In un campo magnetico le righe spettrali di un atomo si scindono in più componenti (effetto Zeeman); nei casi più semplici, osservando lungo la direzione delle linee di forza del compo si nota una scissione in clue componenti di lunghezza d'onda spostata $d \mathrm{i} \pm \Delta \lambda$ da quelli $\lambda_{0}$ delliz riga indisturbata, polarizzate circolarmente l'una in ver'so opposto all'altra (effetto longitudinale); osservando invere perpendicolarnente alle linee di forza del campo, si ha una seissione in tre componenti lineamente polarizzate (effetto trasversale); la componente centrale ha la stessa lunghezza

${ }^{*}$ ) Questo lavoro costituisee la seconda parte del capitolo 5. I primi quattro capitoli e la prima parte del capitolo 5 sono pubblicati rispettivamente in "Ann. Geof." 12, 297 e 389 (1959); 13, 85 e 135 (1960); 16, 161 (1963). 
d'onda $\lambda_{o}$ della riga indisturbata, ed è polarizzata parallelamentéalla direzione del campo, mentre le due componenti laterali, al distanza $\pm \Delta \lambda$ dalla componente centrale, sono polarizzate perpendicolarmente a questa. Infini, osservando obliquamente alle linee di forza, l'effetto trasversale si trasforma nel senso che le due componenti laterali risultano polarizzate elliticamente l'una in verso opposto all'altra.

La differenza $\Delta \lambda$ di lunghezza d'onda fra le componenti e la riga indisturbata è data dalla espressione

$$
\Delta \lambda=\frac{e \lambda_{a}^{2}}{4 \pi m c^{2}} g H=C g \lambda_{a}^{2} H
$$

dove $e$ è la carica dell'clettrone, $m$ la sua massil, $\lambda_{0}$ la lunghezza d'onda, $H$ l'intensità del campo magnetico e $g$ il cosidetto fattore di Landé, funzione dei numeri quantici di momento orbitale e di spin, che può assumere valori compresi tra 0 e 3 . Esprimendo nella [5.9] le varie grandezze in unità e.g.s. ( $e$ in u.e.s.), si ottiene per la costante $O$ il valou $4,7.10^{-5}$.

Per la [5.9], dalla misura di $\Delta \lambda$ si può risalire alla intensità del campo magnetico esistente nella regione dove la riga ha origine. Per avere una ideib dell'entità dell'effetto, si noti chè per $\lambda_{o}=6000 \AA, g=3$, $H=1000$ oersted, si ha $\Delta \lambda=0,05 \AA$. Si comprende quindi conce solo per campi molto intensi si possa osservare un effettivo sdoppiamento delle righe; per intensità piuttosto basse si nota soltanto un allargamento, e in tal enso la misura è resa possibile solo estinguendo a mezzo di opportuni analizzatori l'una o l'altra delle due componeriti.

5.5.2. Campi magnetici delle macchie. - Le macehie solari sono centri di intensi campi magnetici. Tá scoperta risale al Hale (1908), il quale fu condotto alla ricerca di tali campi dalla struttura della cromosfer'a attorno alle resgioni delle macehie, struttura che lo avera fatto pensare alla esistenza di linee di forza magnetiche. Molto più tardi Iale e Nicholson (1938) pubblicarono i risultati di molti anni di studio, dai quali essi e numerosi altri Autori hanno potuto trarre varie condusioni.

I campi magnetici delle macchic sono generalmente piuttosto intensi, dell'ordine delle centinaia o delle migliaia di oersted. Esiste una certa relazione tra l'area delle macchie e il valore massimo $H_{m}$ del campo 
magnetico da esse presentalto: secomblo Houtgast t: Van Sluiters (19-48) tale relazione è esporessa dalla formula

$$
H_{m}=3700 \cdot A(A+66) \text { ocrsted }
$$

dove $A$ ì l'area espressa in milionesimi dell'enisforo visibile. Nicholson (1933) aveva precedentemente dedotto ma relazione dhe dava per $H$ valori un po' minori della espressione suddetia. Fatmanmente si tratta di relazioni empiriche che forniscono valori medi dei campi, i quali possono a volte raggiungere valori bren più clevati; sono stati misurati infatti campi fino a t500 oerstert.

In realtà il valore $H_{m}$ che viene affeltivanente osservato presenta una sistematio dipentenza dalla posizione della macehia sul disco solare: esso è minore per le narehio presso il bordo che per quelle vicine al centro. Ciò potrebbe indienre una dimimzione del campo con l'altezza nell'atmostela solare al di sopra delle marchice, ma potrebber anche trattarsi solo di un effetto apparente, rovuto alla influenza perturbatrice della scintillazione a della diffusione della lue cotosferiea

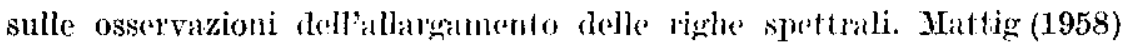
sembra attribuire l'elferto a cuestat sola calusa perturbante.

Il rampo magnetico è massimo al centro dellat malechia, e va diminuendo fino a seompatire al bordo dedla penombra; inoltre, al centro, esso è diretto radialmente al sole, cioé perpendicolarmente alla superfieie solare, ana l'amizolo tra la direzione badiale a il campo va progressivanente aumentando qualudo di si allonlana dal contro, lino a dag-

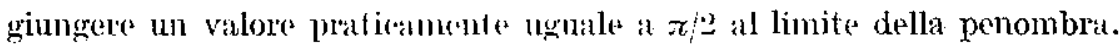
Secondo Broxson (1912), la relazione tra l'intensili del eampo $Z$ e la distanza $r$ dal centro di una macehia considerata approssimativamente circolate, il lagrogio dellat coni penombra sia $b$, is

$$
H=H_{m}\left(1-r^{2} / b^{2}\right)
$$

Questa relazionr viene conf $f^{2}$ mata da Thiessen (1953), il quale gindica inesatta l'espressione di Mrattien (1953).

$$
H=H_{m}\left(1-r^{4 / / 6}\right) e^{-2 r^{2} / b^{2}}
$$

in quanto nel dedurre quest'ultima non si sarebbe tenuto conto di un sistematico effelto ot tico nelle osservazioni, tale da dare valori troppo bassi per l'effetto Zerman a quindi per l'intensità del campo. In ogni caso formule del tipo ore citato lappresentano semplicemente un andamento medio del campo tuagnetico allinterno di una machia; in 


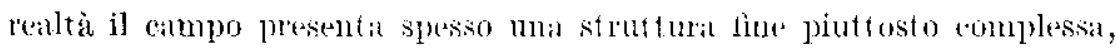
rome estato mesiso in rilievo da severny (1965 b).

Il flusso magnetjere attraterso lanea di una matechia è dato da $\Phi=/ H_{\perp} d \sigma$, dore do è lelemento di supertioriz; noto $H_{m}$. $\Phi$ si può

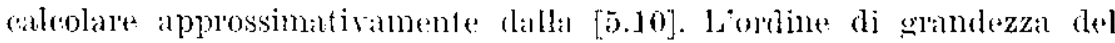

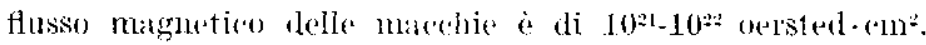

Attiaverso l'esame delle osservazioni di Monte Wilson, Cowling

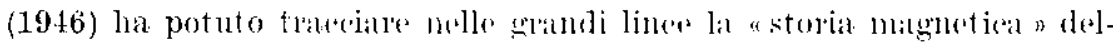
le maechie solari. Durante i primi gioneni della formazione della matcelial

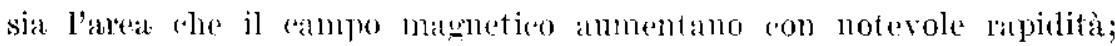

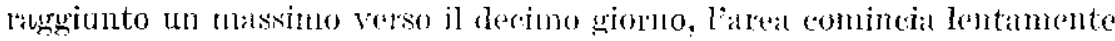
al diminuile, mentre if eampo magmetico rimane pressoché (ostante fin verso la fine della vita dellat materbia, fitambo anche esso diminuisce rapidamente. In termini di flusso, si ha pereceo un notevolissimo aumento iniziale, dovuto all'incemento rontemperaned des campo e chell'ades, seruito da ma diminuziome pratinamente proporzionale alla diminuzione dellarea, e intine vin ato rapido fino alla estiozione.

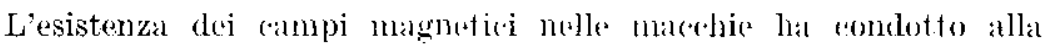

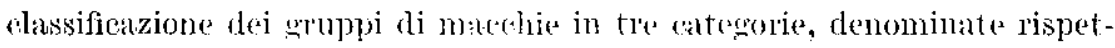
tivamente $\alpha, \beta, \gamma$. T gruppi $\alpha$ hanno un carattre mijolale, diò i loro rampi magnetis:i presentano un solo polo; i gruppi $\beta$ sono bipolari, costituiti, nei casi più semplici, dia che macehit di polarità opposta, ma in generale da più machie, di cui quelle situate nellar regione ovest

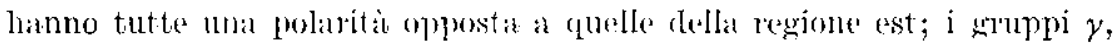
infine, presentano amberlae le polarita, mat distribuite in modo assati irregroline.

Per farsi unt idea della frequenza relativa delle tre ategorie, si noti che dolla statistial di Riclatrolson (1948) risulta che su 6387 gruppi di macehie misumti a Monte Wilson tra i] 1917 a il 1946 ben 5814 , cioè il 91,0\% erano bipolari; 24, pari allo 0 , 4\% erano di tipo $\gamma$, mentre 549 $(8,6 \%)$ erano unipolati. Si conclude quindi che la grandissima maggioranza dẹi gruppi prescenta una regolare bipolarità; ed è inollere da

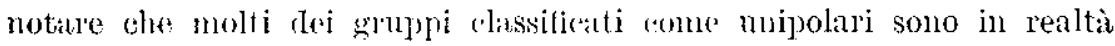
il residıo di erruppi bipolari ben sviluppati; essi sono spesso accompagnati da una regione facolare presentante un notevole campo magnetico di polarità opposta nella posizions! in cui doverebbe trovarsi la macehia principale ormaj dissolta ("mauthe invisibili", secondo la demominazione di Hale).

Un femomeno molto importante è quello roto sotto il nome dj legge della polarita: tutte le monconic ovest dei mruppi bipolari di un certo 
('misfero solare (nord o sud) hamo la stessa polarità, opposta nei due emisferi; tale polarità ambia segno all'inizio di ogni nuovo riclo solare. Dal punto di vista magnetico si ha dunque un ciclo non più di 11 anni, ma di 22 amni. Nel etelo or or's (hiuso, iniziatosi nel 1954, le macchie ovest avevano polarità $N^{r}$ nellemistero nord del Sole, polarità $S$ nell'emisfero sud. Le eccezioni a questa legge della polarita sono molto poche: Richardson ha trovato infatti che dei 5814 gruppi bipolari di rui si è detto sopra solo 180 , cioè il $3,1 \%$ non suguivano bale lexge.

5.5.3. Campi magnetici fotosferici. - Il costante progresso nella tecnica di osservazione dei campi mannetiui solari, culminato nel cosidetto "magnetogralo solare" ideato da Babcock $(1953,1955)$ e perfezionato specialmente da Serelny $(1962,1964)$, hat permesso di stadiare lo stato magnetieo di tutta la superficite solares a non solo delle regioni delle macrhie.

Nella fascia di latitudini eliografiche +500 asistono numerose regioni caratterizzate da campi magnetei loralizzati. Tali regioni non sono permanenti, ma hanno unit vita che va da cirea un'olal a qualche mese; l'intensità dei campi maguetici è meneralmente compresa tra

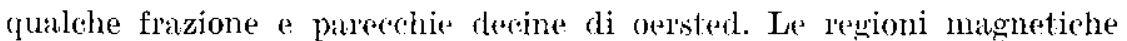
possono classificarsi in due tipi: regioni bipolari (BMI) e regioni unipolari (UM).

Le regioni BM sono le pita numberse; sembra che le machie solari si formino solo in esse, ma cretamente non in tutte. E interessante il fatto che le regioni BM seguono la stessa leurge cli polatità delle marehie in modo quasi rigoroso. Le regioni BXI hamo dimesnsioni assai variabili; le più estese possono giungere a più di un decimo di emisfero visibile. le più piecole non superano il millesimo. Secondo le osservazioni dei Babcock vi ì una stretta colrispondenza tra rescioni caratterizzate dil forte emissione eromosferica nella risa $K(\lambda=3133,7)$ del CaII (facole) e intense regioni BN.

Con un magnetogralfo di Bubcock modifuesto, Howald (1959) hat misurato, nell'autunno $195 \pi$ e mell'estate 1958, (ioè al massimo dell'attività solare, i campi magnetici in varie regioni di macehie, trovando che molto spesso presso le machie ma al di fuori di esse esistono rampi maggiori di 75 oersted (limite (i linearità dello strumento); cio naturalmente porta a modificare le espressioni del tipo della [5.10] sulla distribuzione del campo. Un confronto con yli spettroliogrammi in CaII conferma la corrispondenza già trovata dai Babcook tra campi magnetici e facole cromosferiche; più esattamente, secondo Howird il confine 
delle facole coincide con buona approssimazione con la linea di eontorno di 10 oersted. Una ulteriore conferma di ciò è data dalle osservazioni di Leighton (1959), il quale, dit misure eseguite nell'agosto e settembre 1958, conclude che vi è uno stretto accordo fra la forma delle facole in luce di CaIT e quellat delle regioni magnetiche, e che in corrispondenza delle facole il campo magnetiro raguiunge spesso valori di $100 \div 200$ oprsted.

Secondo Simon (1963) tale stretta correlazione si estende fino ai campi magnetici più deboli (cirea 2 oersted). Sembra inoltre che l'intensità del campo presenti una notevole correlazione con l'intensità di emissione delle facole, cosicché una l'appressntazione dellat struttura cromosferisa potrebbe venir considerata come la milpa solare del valore assoluto del campo magnetico (Lejghton, 1965).

Le regioni UM sono zone, nemetalniente molto estese, della superficie solare in eni si osserva un canpo masnetico di una unira polarità. L'intensità del campo è bassi, cha frazioni cli oersted a circa 3 oersted.

Malgrado siumo meno numerose dele regioni BM, le regioni UM non sono tutiavia dei fenomeni rari. Scoperte dai Babeock (1955) durante la fase di declino del cielo solale (1952-54) e considerate alloral come eventi di curatere piuttosto eccezionale, esse sono state nuovamonte osservate da Howard (1965) nel periodo compreso fra l'estate del 1959 e la fine del 1962, in ura fase del ciclo solare praticamente corrispondente a quella analizzhta dai Babeock. Howard ha studiato piu di 100 regioni UM di notevoli dimensioni; in esse il campo magnetico non è distribuito con continuità, ma è concentrato in piccole zoní. Contrariamente a quanto si pensava dopo le prime osservazioni, anche la regroni UN sono associate d manifestazioni cromosferishe; infatti, secondo Lowasd, wli spettrogrammi di caleio presentano, in corrispondenza di una regione UM, un amento della emissione di fondo.

Le regioni UM si formano dalla disinteytazione di regioni BM ben sviluppate nell'ultima fase di esisternza di gueste. Dopo un tempo variabile a partire dalla loro formazione, ma dell'ordine di qualche mese, queste regioni BHI si uspandono, con diminuzione di oumpo, e infine si disintegrano, lasciando due regioni UM di polarità opposte, che tendono a migrare in direzione dei poli. Ia vita delle singole regioni UM può essere a sua volta notevolumente lunga: la più importante delle regioni unipolari osservate dai babcock è durata almeno sei rotazioni solari, dal maggio all'ottobre 1953 (v. anche 1.5.5.1.).

Nella valutazione di questi risultati oceorre tuttavia tener conto di un fatto molto importante, messo in luce da Severilly (1965 a): aumen- 
tando il potere risolutivo dello strumento, unla l'egione unipolare può apparire magneticamente molto più complessa, con strutitura multipolare.

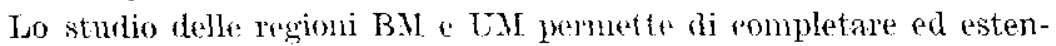

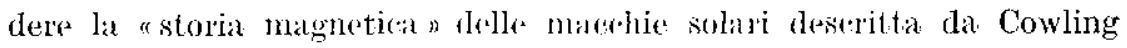

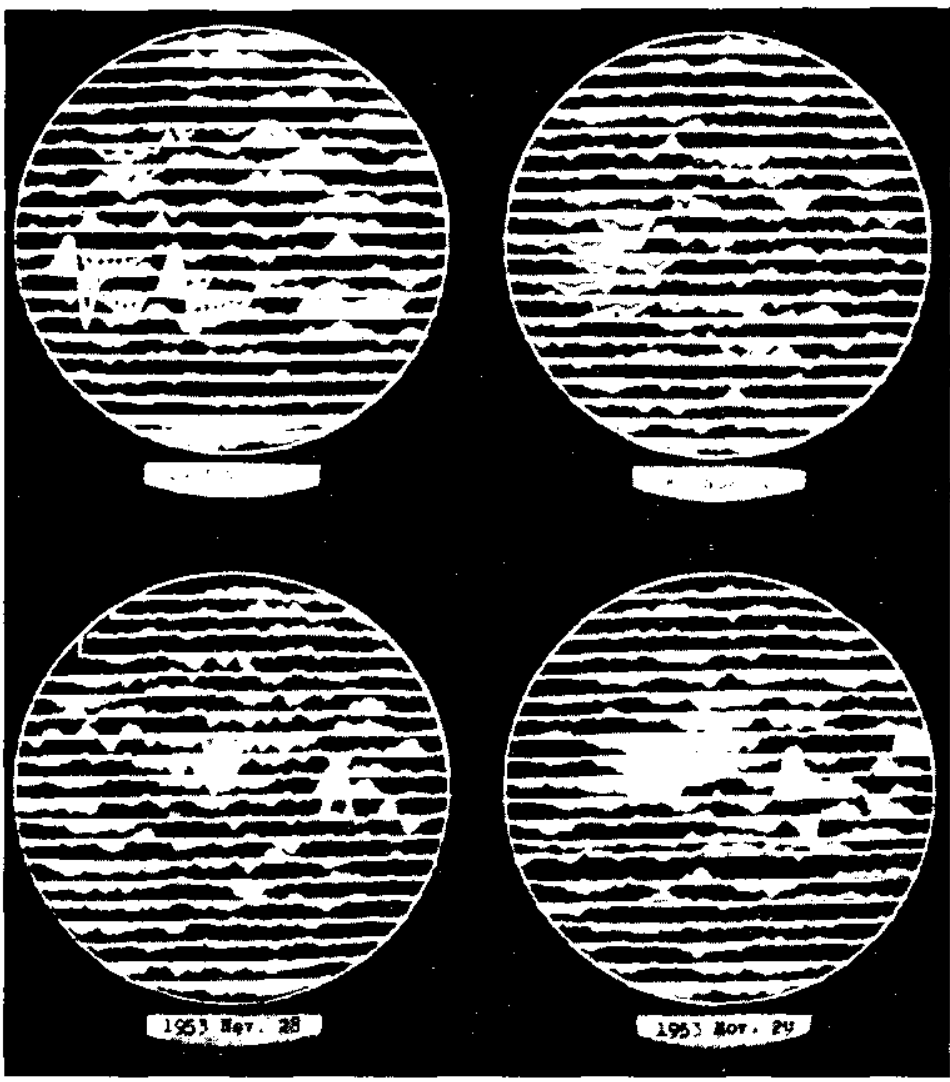

Fig. 5.37 - Esempi di mignetogrammi solari ottenti da Babeock: sono visibili regioni B.I e U.I, nomehe pularita opposte e pinttosto miformi nelle dhe calotte polari (secondu Babeock).

(v. 5.5.2); esso anzi suggerisce dhe il fenomeno fondimentale dell'attività solare sia la vita e l'evoluzione dello regioni manetiche, mentre macchie, brillamenti e facole non sarebbero che episodi di tale evoluzione. Infatti le regioni magnetiche nascono qualche ziolno prima delle macchie e delle facole, durano per molto tempo dopo the queste manifestazioni sono scomparse, e rappresentano infine l'estremo residuo dei centri di attività. 
5.5.4. Brillamenti e campi magntici fotosferici. - E interessinte studiare quanto avviene nei campi matumetici osservabili nella fotosfera, nelle regioni al disopera delle quili hamo luogo i brillanenti. Importanti informazioni sono formite dall" osserrazioni di Gopasyluk a coll. (1963),

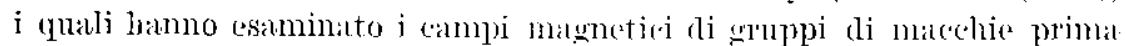
0 dopo 52 brillamenti di valia importanza fra il luslio 1955 e la fine del

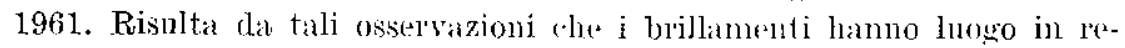
gioni dove eststono forti gradienti manetici: limportanza del brillimento cresee al crescere della intensita dei erradienti. In seguito al brillanento i gladienti diminuiseono nettamente, in misura tanto masgiore quanto magriore è l'importanza del brillamento stesso.

Alcuni casi particolari, stuliati in modo dettagliato, haluno fornito ulteriori informazioni. Ar essempio, Ie osservazioni combinate di Howard e Severny (1963) a Honte Wilson a all Osservatorio Astrofisico di Crimea in occasione del brillamento di impertanza $3+$ del 16 luglio 1959 hanno mostrato che in seguto ad esso si è verificata una variazione non solo dei gradienti ma anche della intensità del campo, che è diminuito di un fattore 3; la diminnzione di energia magnetica è stata valutata a circa $10^{32}$ erg. E interessinte il fatto che in realtà solo i campi magnetici di valore elevato sono stati modifinati in tale evento; le osservazioni di Monte Wilson, riportate da Howirl a Babcocli (1960), hanno infatti mostrato che per i valori non superiori a 10 oersted (limite superiore dello strumento usato) i campi magnetici fotosferici nella regrione del brillamento non hanno presentato dulante il fenomeno variazioni degne di rilievo; anche la struttura filanentosa comosferica attorno al brillamento, indisatrice di linoe di forza matsmetiche nella cromosfera, è rimasta pressoché invariata.

Variazioni piuttosto complesse nella configurazione dei campi magnetici sono state osservate da Severny (1965 ( $)$ in oceasione del brillamento di importanza 2 del 22 giumno 1962; in questo caso non si può parlare di una rapida diminuzione del ampo in semerale, ma piuttosto di forti leformazioni locali.

5.5.5. Campi magnetici nella cromosfera e nella corona. - I campi di cui si è parlato nei numeri precedenti sono quelli esistenti nella fotosfera, e sono i soli cumpi magnetici solari per i quali è possibile la misura diretta. I naturale supporre che i campi fotosferici si estendano anche al di sopra della lotosfera, nella cromosfera e nella corona.

Questa supposizione è confermata da osservazioni di vario tipo, in primo laogo da quelle del movimento di materia risadente dalle prota- 
beranzo solari (osservate al bordo) nella fotosfela. Essendo la material delle protuberanze fortemente ionizzata, essa tenderà a seguire, nel suo moto, le liner di forza di eventuali ("ampi. (orrell e coll. (1956, 1958), studiando i moti della materia di die protuberanze presso il bordo del Sole il 26 febbraio 19.16 e il 20 :1prile 1950 , hamno concluso che tali moti avvenifano sotto l'aziont di un campo di clipolo, il cui centro era rispettivamente 0,2 e 0,03 laygri solari al di sotto della superficie. La protuberanza del 20 aprile 1950 si è prodotta al di sopra di un importante gruppo di macchie, nel quale la massina intensita del ampo magnetico aveva raggiunto i t200 oes'sted. Le tratettorie del moto della materia, supposte coincidenti con le lines di forza del ampo magnetico, si estendono fino a cirea $7 \cdot 10^{4} \mathrm{~km}$ al di sopma della fotosfes. I quindi evidenta che i rampi delle macrhite si testendono a notevoli altezze nella aromosfera; essi giungono anche nella colona, come induce a pensare la formal degli arohi coronali al di sopra dei eentri di attività. A quest'ultima conelusione si perviene anche in base alla osservazionta "he fome vedremo più estesamente in 5.6) la l'adioemissione solare di linghezza d'ondat attorno al metro, che ha certamente una origine colonale ed è pasticolamente associatal ai centu di attivita, e weneralmente polarizata, indicando l'esistenzal di notevoli ("ampi matgnetie.i.

A questo proposito è interessante uno studio di Cohen (1961) su di un fenomeno di ladiopropagatzione (e quindi indipendente dalla natura della sorgente) nellatmosfera solare: in molti busts di radioemissione nella gamma delle microonde si osserva una polarizzazione circolare, in cui il senso di rotazione si inverte a una certa frequenza. Questo fenomeno, interpretato in termini a terolia magmetoionis"s, slggerises che il campo magnetico ad una altezza di cil'ca $10^{5} \mathrm{kml}$ al di sopra rli una regione attiva ha an valore compreso fra 206 oersted.

5.5.6. Campo magnetico gencrale del Sole. - Una questione di grandissimo interesse è infine quella defla esistenza o meno di un campo mignetico generale del Sole, campo dhe, in analogia con quello terrestre, potrebbe in linea di principio essere approsisimato con un cumpo di dipolo.

Tale ipotesi sembra confermata dalla forma dei raggi coronali polari, che si possono osservare verso il minimo della attività solare; essi hanno infatti l'aspetto delle linee di forza di un dipolo, il cui asse coincide upprossimativamente con l'asse di rotazione solare. Misure accurate eseguite in occasione di varie eclissi hanno mostrato che in realtà i raggi divergono in misura maggiol' $\mathfrak{i}^{2}$ di quanto fanno le linee di forza 
di un dipolo, se semblat anche che tale divergenza vari dulante il dido solare (van de Hulst, 1953).

I primi tentativi di misura diretta del ampo magnetico penterale del Sole furono opera di Hale e dei suoj collaboratori (IIale, 1913: Seares, 1913; Hale e coll., 1918). Essi conclusero the il "ampo solare è un cimpo di dipolo, il cui asse è inclinato di pocthi gradi rispetto all'asse di rotazione, e il cui momento milgnetive ammonta a $8.4 \cdot 10^{33}$ oersted $\cdot\left(\mathrm{m}^{3}\right.$, il che comporta pere il ampo polare lin valore di jo oersted: l'orientazione del dipolo rispetto allasse di rotazione is analoga a quella terestre, ciò al polo sliografíco nord corrisponde una polarita sud e viceversa.

Successive misure eseguite in amsi più recenti con dispositivi sempre più perfezionati da von Klüber e da fhiessen non hamo tuttavia confermato tali andusioni; l'intensità del ampo polare è risultata di soli pochi oersted, con indicazioni rhe l'orientamento dell'asse del dipolo c̀ opposto a quello del dipolo terrestre.

Il magnetografo solare di Babcork ha fornito un ulteriore importante contribute alle osservazioni lel campo magnetico generale del Sole (Babeock, 1955). Secondo le osservazioni aseguite negli anni 1952-54 tale campo è rilevabike solo a latitudini eliografiche maggiori di circa \pm 550. L'infensità della componente nella direzione di osservazione è, in vicinanza dei poli solari, rirea 1 ocrsted assumendo come direzione delle linee di forza in tali regioni quella dei raxgi coronali, si può antmettere che il valore medio dell'angolo tra le linese di forza e la direzione di osservazione sia attorno ai $60^{\circ}$, da a risulta per il campo polare un valore delloordine dei 2 oersted. Ia polaritic é risultata opposta a quellat del dipolo trerestres.

Sono estremamente interessimti te misture eseguite in seguito. Infatti, sempre secondo Babrock (1959), tra il marzo e il luglio del 1957 il campo nella regione del polo eliografieo sud ì dapprima dininuito di intensità fino ad annullarsi, poi ha rambiato rerso; si sono così avute due polarità dello stesso sexno (norel) tino al novembre 1958, quando quasi improvvisamente anche il empo della alotta nord ha cambiato polarita, ricostituendo in tal modo il dipolo, questat volta orientato come" quello terrestre.

Dalle misure di Howard (1965) a Monte Wilson, eseguite con continuità a partice dal 1960, risulta confermata la nuova polaritì del campo generale del Sole, almeno fino al marzo 1961; a questa epoca l'intensità del campo nei pressi del polo sud solare è diminuita fino a risultare praticamente nulla, e tale sembra essere rimasta fino all'estate del 1963, chata della comunicazione di Howard. E di quest'ultima epoca 
una osservazione di sererny eflettluata con un potere risolutivo assai

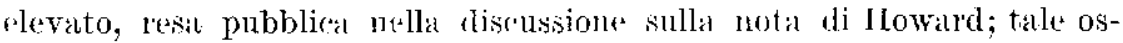
servazione conferma sostanzialmente quelle di Howald, precisindo tuttavid the al polo sud solare si nota vano zone di ampo positivo in numero ugurte a quelle di campo nenativo, a questa ela la lagione pere eni il risultato dava in media intensita mulla. Dalle osservazioni di Howade

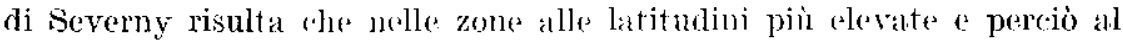
di fuori della fascia dej centri di attività, a quindi capaci di fornire informazioni più chiare sul atupo generalk del Sole, il campo magnetico non è distribuito uniformemente sulla fotosfara, ma è concentrato in piccola aree, the in pration coincidono con i flocenli polari in luce di CaII; si tratta della stessa cardteristioa ossorrata, come abbiamo visto, da Howard nelle regioni unipolari.

E importante notale whe serife liserve song state sollevate (Alfvén, 1956; Alfvén e Lehnert, 1956) sulla attemdiljilita della deduzioni attorno a deboli campi magnetici solari falte sulla batse dell'effetto Zeeman. Secondo Alfvén, la turbolenza della fotosferal pivelata dalla presenzal della granulazione deve produre un ampo magnetico turbolento di alenne centinaia di oersted; la misura dell'tlletto Zeeman viene necessariamente eseguita, a catusa dei limiti della risoluzione strumentale, su di una regione fotosferia contenente migliaia di ghanuli e nelle conchnsioni fin qui tratte si esupposto che il risultato dessesemplicemente un valor medio del campo magnetieo in questa rexgione. Ná non è detto che l'influenza del campo turbolento sia in modia nulla, poiché esso deve essere necessariamente "accoppiato" con la densiti a la temperatura, dalle quali dipende l'assorbimento di ma riga dello spettro, e puó darsi che il modo di aceopriamsuto sia tal: la falsilte sistematicamente la misura del campo basata sull'pffecto Zeeman. In assenza di una teoria esatta dell'effetto Zeeman in condizioni fisiche quali quelle della fotosfera non è possibile, afferma Alfvin, litenere conclusive le misure su di esso basate.

$A$ questa interessante obiezione si possono tuttavia contrapporre due dati di fatto: 1) i deboli campi delle lectioni bipolari obbediscono alla legge di polarità, come abbiamo visto in 5.5.3., à è un forte argomento a favore dellit attendibilità dell'uso dell'afl'et to Zeeman; 2) le misure della polarizzazione delle componenti delle jighle usate proverebbero anch'esse che si tratta di un autentico effetto Zeeman.

E tuttavia necessirio tener conto dell'influenza del potere risolutivo dello strumento sui risultati, influenza messa in luce soprattutto da Severny. Agli effetti del campo magnetico generale del Sole sono da 
eitare a questo proposito le misure di Stenflo (1966) col magnetografo dell'Osservatorio di Crimea con fenditure di varia grandezza, dalle quali risulterebbe nelle regioni polari del sole mat intensit i del campo crescente al diminuire delle dimensioni dilla fenditura e quindi all'amentare del potere risolutivo, intensità the salrebbe dell'ordine dei 5 oetsted, con una polarità parallela a quella dol campo tererestre.

\subsection{Radioemissione solare.}

Dar quanto detto nei preecedenti eipitoli, in particolare in $\mathbf{1}$ e $\mathbf{2}$; e nella prima parte del pressenter capitolo, è chiaro che l'ipotesi joù immediata riguarko alla causa prossima delle tempeste matgnetiche e fenomeni concomitanti d̀ quella di una nuvola di particelle emessa dal Sole; per la mategoria delle tempeste s.c. tals emissione dovrebbe aver luogo con carattere asplosivo durante un brillamento. La forte influenza. del campo geomagnetico sul corso \& sulla distribuzione delle perturbazioni, nonché la produzione dei fenoneni aurorali, randono inoltre evidente che si tratta di particelle elettricamente cariehe.

Questa ipotesi apre orviamente notevoli problemi sulla natura delle particelle, sul processo di amissione a sulla loro propagazione nello

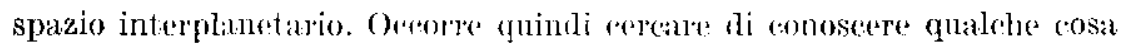
di più preciso ste tale ladiazione corpuscolates.

A questo scopo puó servire sist una eventuale osservazione diretta della nurola di particelle (ariche (plasma) nel tragitto dal Sole allat Terral, sia, con procedimento più indiretto ma di più facile disponibilità, lo studio approfondito della comnessionse tra i fenomeni solari e gli eventi terrestri. Iniziamo da questo secondo procerdimento, passando in rassegna i principali risultati ottenuti negli nttimi anni, dopo la pubblichzione del apitolo 1 . E rome primo passo illustreremo brevemente le nuove conosecnze arquisite sui fenomeni di radioemissione solare, limitandoci tuttavia alla cosirletta ralioemissione de Sole attivo, fenomeni che si sono dimostrati di grandissina importanza nello studio della interdipendenza fra attivita solare ed erenti greofisici.

5.6.1. Henomeni di radioemissione turante un brillamento. - In 1.5.4.3. e 1.5.4.4. abbiamo messo in evidenza che una caratteristica fondamentale di un brillamento solare produtivo di tempesta magnetica è la esistenza di iutensul rudioemissione, ed abbiamo accennato alla esistenza di varie specie di radioemissiome, aratterizzate ciascuna da un 
particolare aspetto dello spettro di frequenza (bursts di tipo II, III e IV). Riprendiamo qui l'argomento, descrivendo in modo più particolaregriato quanto si è fino ad ougri scoperto su questi tipi di radioemissione.

5.6.1.1. Outbursts di tipo $I I$. - Sono compresi in questa categoria gli outbursts, della durata dell'ordine dei 10 minuti, costituiti da una intensa radiazione in una banda piuttosto ristretla di frequenze, banda che si sposta lentamente verso le frequenze più basse (slow-drift bursts). In questo spostamento, grli outbursts spazzano tutte le gamme d'onda

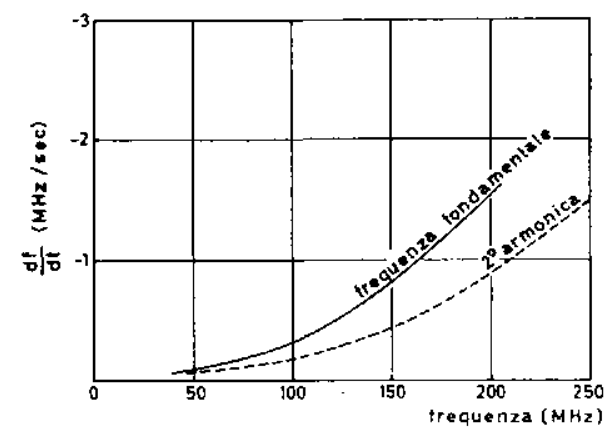

Figr. 5.38 - Deriva di frequenza legli outbursts di tipo II (secondo Maxifell e Thompson).

dalle onde centimetriche alle onde decametriche (ciò̀ da circa $10^{4}$ a $10 \mathrm{MHz}$ ); passando dalle alte alle basse frequenze il fenomeno si presenta con una intensita rapidamente crescente: mentre alle onde centimetriche l'intensità dell'outburst è dell'ordine del 5-10\% del livello di base, essa sale al 100\% alle onde decimetriche, a un valore di $10-10^{3}$ volte quello del livello di base alle onde metriche, a addirittura a un valore $10^{6}$ volte magriore alle onde decametrichc. La temperatura equivalente (temperatum di un corpo noro che emette su tali frequenze con la data intensità) giumge ad un valore dell'ordine di $10^{10}-10^{11}{ }^{\circ} \mathrm{K}$; ciò rende evidente che la cadioemissione di tali outbursts non può essere di origine termica.

La deriva di frequenza è stata studiata da Maxwell e Thompson (1962) su sessanta eventi fra il 10 ottobre 1950 e il 31 dicembre 1960 ; essa appare come una funzione crescente della frequenza (fig. 5.38), con df $/ d t \cong-0,3 \mathrm{MHz} / \mathrm{sec}$ attorno ai $100 \mathrm{MHz}$, e- - 1,5 MHz/sec sui $200 \mathrm{MHz}$.

Una caratteristica interessante degli outbursts di tipo II è che gran parte di essi (circa l'80 per cenio secondo Maxwell e Thompson) sono 
costituiti da una ladionmissione su di ma frequenza fondumentale ancompagnata da una seconda amonica, whe presenta una intensita praticamente uguale a quella della frequenza fondamentale. Anche questo fatto porta ad escludere ele la ladioemissione in estme sia di origine termicur.

Gli outbursts di tipo II presentano una strettissimal associazione con i brilamenti, in particolar modo con quelli piu importanti, nel senso che essi sembrano verifian'si solo in orcasiono di questi; solo porhi sono stati osservati senza che alem brillamento renisse segnalato, ma non è chiaro se eio sia dovuto solo a maneanza di osservazione of tical solare.

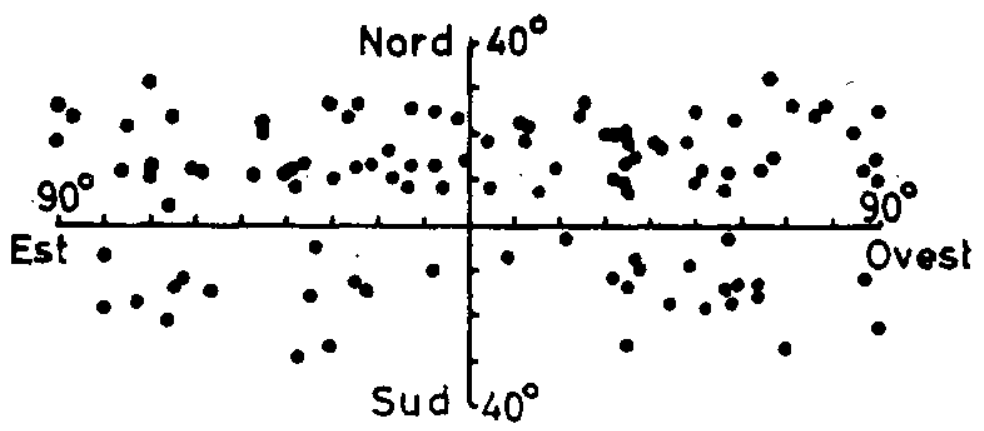

Fir. 5.39 - Distribuzione eliograficia di brilkamenti issociati con outbursts di tipu Il (secombo Maxwell e 'lhompson).

in quelle occusioni. In fig. 5.39 i riportata la posizione in latitudine e longitudine eliografiche di 106 brillamenti associati ald outbursts di tipo IT osservati da Haxwell "Thomysom (1962); tali brillamereti sono ugualmente distribuiti su tutte lo longitudini, il alu rivela che lat radiazione di tipo II viene emessa in coni molto larghi.

L'interpretazione più attendibile desli outbursts di tipo II sembra essere quella che attobuisee tale malomissione a oscillazioni di plasma nella cromosfera e nella corona solare. F noto infatti the in un gas ionizzato, soggetto ald una rapida perturbazione, si possono produrre oscillazioni con una frequenza fondamentale

$$
f_{n}=\sqrt{N e^{2} / \pi m}
$$

dove $N$ è la densità elettroniea in $\left(\mathrm{m}^{-3}\right.$, e è la carica dell'elettrone in u.e.s., $m$ la massa dell'elettrone in gr; al cossare della perturbazione, l'ampiezza delle oscillazioni diminuisce esponenzialmente con una costante di tempo pari a $1 / v$, dove $v$ è la frequenza di collisione tra elet- 
troni : ioni positivi. Una frazione della energia delle oscillazioni del plasma si trasforma in radiazione clettromannetica. Aggiungiamo che secondo Westfold (1949) in presenza di un rampo mignetico $H$, oltre alla frequenza di oscillazione $f_{0}$, si possono produrre le frequenze

$$
\begin{aligned}
& f_{1}=\sqrt{f_{o}^{2} ;}\left(f_{H} / 2\right)^{2}+f_{H} / 2 \\
& f_{2}=\sqrt{f_{o}^{2} \div\left(f_{H} / 2\right)^{2}}-f_{H} / 2
\end{aligned}
$$

dova $f_{B}$ è la girofrequenza per gli elettroni, data da

$$
f_{H}=e H /(2 \pi m e)
$$

A questo fatto potrebbe essere dovuta la struttura complessa della componente fondamentale e della armonica di molti outbursts di tipo II, con la loro suddivisione in due sottocomponenti (Wild 1950; Maxwell e Thompson, 1962). Ciò permetterebbe di determinare l'intensità dei campi magnetici alle varie altezze solari, che strebbero dell'ordine di qualche oersted (v. 5.5.5.). Roberts (1959) ha pesò avanzato molte obiezioni a tale interpretazione.

Come si ̀̀ detto, l'intensità della radiazione plettromagnetica decade esponenzialmente con una costante di tempo $1 / v$. Secondo Smerd e Westfold (1949) (v. anche Pawsey o Smerd (1953)) lat frequenza di collisione degli elettroni ̀̀ data da

$$
v=\begin{aligned}
& +e^{4} \pi^{1 / 2} Z^{2} N_{i} A \\
& 3(2 m)^{1 / 2}(k T)^{3 / 2}
\end{aligned}
$$

dove $k$ c̀ la costante di Bollzman, $Z$ la carica ionica in unilà di carica elementare, $N_{t}$ la densità degli ioni positivi ed $A$ la funzione

$$
A=\ln \left|1+\left(\frac{ \pm k T}{Z e^{2} N^{1 / 3}}\right)^{2}\right|
$$

Assumendo una atmosfera solare costituita da idrogeno completamente ionizzato, avremo $Z \rightarrow 1+N_{i}=N$. Per una frequenza di oseillazione di $200 \mathrm{MHz}$ e per $T=10^{6} \mathrm{oK}$, si ha, dalle [5.11] $\mathrm{a}$ [5.12], $v=$ $27 \mathrm{sec}^{-1}$; per una frequenzal di $20 \mathrm{MHz}, v \approx 0,27 \mathrm{sec}^{-1}$. Ciò significa che l'oscillazione si smorza in tempi dell'ordine del secondo al cessare della causa eceitatrice.

Cone è noto, la frequenzat fo ènche la frequenza critica per una data densità elettronica $N$ : l'indice di rifrazione per un'onda elettro- 
magnetica di frequenza $f_{0}$ diviene, per quella datal $N$, uguale a 0 , mentre per una densità superiore esso diviene immaginario. Se si ammette the la densità elettronica della cromosfera e della corona diminuisea con I'altezza, dalla reguion sottostante alla data densità ${ }^{*}$ non può sfuggire quindi alcuna radiazione alettromagnetica di frequenza nuale $o \mathrm{in}$. feriore a $f_{0}$.

La deriva di frequenza dexli ontbursts di tipo II fa pensare che essi siano dovuti a oscillazioni di plasma in revioni di densità elettronica via via minore, ciò̀ ad altezze via vial maggeriori. Lal sorgente che perturba il plasma si sposterebbe quindi verso laalto. Conoseendo la variazione di $N$ con l'altezza, dalla deriva di frequenza df dt si può dedure la velocitì della soresente.

Ponendo $Q=r / R_{o}$, dove $r$ è la distanza dal rentro del Sole e $R_{0}$ il ragrgio della fotosfera, una forma attendibile della funzione $N(\varrho)$ nella corona solare, è data dalla espressione di Bambach-Allen (Allen, 1947).

$$
N=10^{8}\left(1,55 \theta^{-8}+2,99 \varrho^{16}\right)\left(\mathrm{Im}^{-3} .\right.
$$

F da notare tuttavia che tale modello si riferiscer ad una corona non perturbata, mentre co noto che in corrispondenza dei centri di attività la corona presenta delle comdensazioni, nelle quali la densita elettronica può aumentare di un fattore 10 rispetito ai valori dati dalla [5.13]. In effetti Maxwell e Thompson (196:), utilizxando numerose misure interferometriche dellaltezza delle sorgenti di ralioenissione sopra centri di attivita a varie frequenze, trovano she la distribuzione della frequenza (cioè della densità $N$ ) in alterza si acororda loenc', almeno per frequenze inferiori a $500 \mathrm{MHz}$, con un modello di rorona in cui $N$ è data dalla [5.13] moltiplicata per 10.

Con questa motifina della [5.13], da essit a dalla [5.11] si deduce la velocità rudiale $c_{r}$ della solgente della perturbazione:

$$
v_{r}=--4,9 \cdot 10^{-3} \frac{\left(1,50+2,99 Q^{-10}\right)^{1 / 2}}{9,3 Q^{-4}+4 f} d \frac{2,8 Q^{-14}}{d t} \mathrm{~km} / \mathrm{secc}
$$

Dai valori di dfid dedotti dalla fig. 5.38 si rieava la velocità ladiale in funzione della frequenza (o dell'altezza), come mostrato in fig. 5.40. Da essa si nota che la velocità è dell'ordiné dei $1000-1500 \mathrm{~km} / \mathrm{sec}$, cioè maggiore per un fattore 3 di quella dedotta in precedenza da altri autori che si basavano sul modello di Baumbach-Allen non modificato.

In uno studio analogo a quello di Maxwell e Thompson, Weiss (1965), basandosi sullo spettro dinamico (deriva di frequenza) di 21 outbursts di tipo II osservati fr'al il 1952 a il 1963, e usando, anziché 
il modello coronale di Baumbach-Allen moltiplicato per 10, il modello di Newkirk (1961) per la densitì elettronica Inngo l'asse di un raggio coronale moltiplicato per 2, giunge a conclusioni non molto dissimili a quelle di Maxwell e Thompson ma con velonità leggermente inferiori; egli fa tuttavia notare l'influenza della scelta del modello coronale sui risultati.

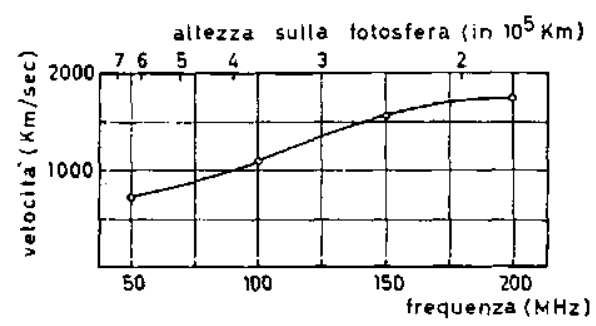

Fig. 5.40 - Velocità radiale media delle perturbazioni origine degli outbursts di tipo 11 (secondo Maxwell e Thompson).

E probabile che le oscillazioni del plasma siano prodotte dal passaggio attraverso la cromosfera te la corona di unir nuvola di partjcelle, verosimilmente elettroni e ioni positivi, in particolare protoni, emessi durante il complesso fenomero the ha come manifustazione ottica più evidente il brillamento. Pjù esuttamente, l'eccitazione delle oscillazioni sarebbe prodotta dal fronte di un'onda d'urto idjomagnetica dovuta al fatto che la velocità della nuvola di palticelle o stuperiore alla veloeità del suono e che la regione in cui l'evento ha luogo, cioè in prossimità di un brillamento, è caratterizzata in generale dall'tsistenza di campi magnetici. L'esistemza della seconda armonies rivela una non linearità nel meceanismo delle oscillazioni. Quanto alla polarizzatziome della padioemissione, essa sombra piuttosto searsa e in ogni caso senza un carattere sistematico.

Mediante misure interteronetriche (Woiss o Slleridan, 1962) sono state studiate anche le dimensioni della sorupente di un oubursts di tipo II; è risultato che il diametro della regione di emissione è dell'ordine dè 10 primi di arco.

5.6.1.2. Bursts di tipo III. - Anch'tssi, come quelli di tipo II, presentano una calatteristica deriva di frequenza, la quale però è molto maggiore che per gli outbursts in precedenza descritti; si ha infatti 


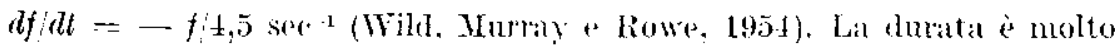
più breve: i bursts isolati possono dmare una deccina di secondi, ma molto spesso essi si presentano in gruppi della durata romplessiva di pochi minuti. Tali bursts sono molto piu frequenti degli outbusts di tipo II. Benche la loro forma sia pir semplice, lit loro intensità è peró dello stesso ordine tanche maguioues, eon una temperatura aquivalente che ragginnge ; $10^{11} \mathrm{oK}$.

Anche i bursts di tipo 1 [I somo probabilnente dornti a oscillazioni di plasma; col metodo precedentemente descent to si puó dedure la velocità della soræente della perturbazione. (he pisnlta compresa fra i 60.000 e i $150.000 \mathrm{~km} / \mathrm{ser}$. Ciò sembral roufermato da misure interferometriche (Wild, Sheridan e Trent, 1959), secondo rui la sorgente si muove con velocità dell'ordine dei $10^{5} \mathrm{kml} / \mathrm{sec}$. L'opinione, espressa per la prima volta da de Jasere (1960), whe si tratti di un fascio di eletroni di tali velocità, sembra ora generalmente areettata (v. ad es. Stewart, 1965; Takakura, 1966).

Per quanto riguarda la polatrizzazione, le osservazioni degli ultimi anni (Komesanoff, 1958; Cohen, 1959; Akabante Cohen, 1961: Bhonsle e MeNarry, 1964; Gopala Rao, 1965) hamno acertato (he i bursts di tipo III sono indubbiamente polarizzati, anche se il srado di polarizzazione $\dot{e}$ in generale moderato.

Come per il tipo II, abche per questa ladiazione le dimensioni de]la sorgente sono dell'ordine dei 10 prini d'areo, e sembra che esse aumentino con l'anmentare dolla lunghezza d'onda (Contrez, 1960).

Una catergoria particolare di bursts di tipo III sembra esseri costituita dai cosidetti bursts a " $\succeq$, cosi chiamati a causa dalla forma dello spettro dinamico (Maxwell t Swarup, 1958). In altre parole, essi presentano all'inizio una deriva di frequenza negativa, approssimativamente della stessa grandezza dei normali bursts di tipo III; raggiunta una frequenza minima, generalmente attorno ai 100-150 MHz, la deriva diventa positiva, con un affld pratiomente uguale in valore assoluto alla prima parte dell'evento. Questo ha nel suo complesso una durata seneralmente minore di 10 secondi. Nella interpretazione data per $i$ bursts di tipo II e III si tratterebbe di nna sorente di oscillazioni di plasma che dapprima sale ad clevata velocità nell'atmosfera solare per' poi ridiseendere. Serondo Maxwell e Swarup (1958) e Hughes e Harkness (1963) questa sorgente seguirebbe le linee di forza di un eampo magnetico fra due regioni di polarità opposta; secondo Takakura e Haddock (v. Takakura, 1966) sarebbe preferibile pensare whe la nuvola elettronica ('messa verso l'alto non abbia in tali casi (al cont lario di quanto avrer- 
rebbe nei tipi III normali) velociti sufficiente a superare i campi magnetici, e dopo aver "piegato" le linee di forza verrebbe respinta verso il basso (fig. 5.41).

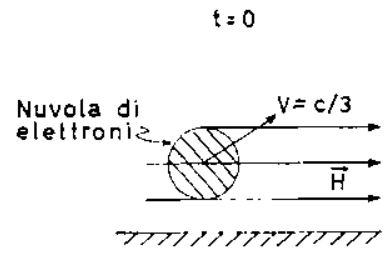

(a)

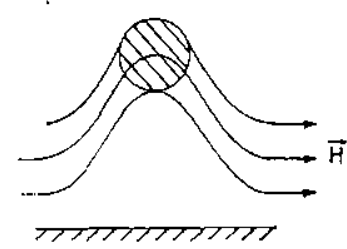

(b)

Fig. 5.4I - Modello di burst di tipo $5:$ in a) inizio del burst e noto obliquo della sorgente verso l'alto; in $b$ ) fase di massima altezza (secondor Takakurat e Haddock).

5.6.1.3. Outbursts di tipo IV. - Come si de areennato in 1.5.4.4., un altro tipo di radioemissione associato ai brillamenti ̀̀ stato individuato da Boischot (195i) mediante osservazioni all'interferonctro di Nançay sulla frequenza di $169 \mathrm{MHz}$, da lui estato chiamato di tipo IV. Si tratta di un forte anmento di flusso della durata di aleune decine di minuti a anche di aleume ore, spesso com una polarizzazione prevalentemente circolare. Questi outbursts di tipo IV sono stati studiati dib molti autori, soprattutto per la loro associazione con importanti eventi geofisici, come vedremo più avanti; si d trovato che essi si estendono in una vastissima gamma di frequenze, dalle onde centimetriche alle onde metriche, e sono sostanziahnente costituiti da mo sjettro continuo di frequenza, di intrusita dell'brdint', ancher maggiore, di quella degli outbursts di tipo II.

Ta grande varietio di aspetti deg]i eventi di radionmissione ai quali, dopo la comunicazione di Boischot, è stata attribuita la denominazione "tipo IV ", ha reso necessario un ateordo per una definizione non troppo vaga e generica. Al congusso Internazionale di Kyoto del settembre 1961 è stata scelta la seguente definizione (Wild, 1962): si assume come burst di tipo IV nn evento di lunga durata e spettro continuo in uua qualsiasi gamma di radioemissione, seguente a un brillamento.

Con questa definizione, l'outburst di tipo IV si presenta in genere in una forma assai complessil. Vi si possono distinguere varie componenti, sia secondo il priterio della sureessione cronologica, sia secondo il eriterio delle caratteristiche fisiche.

Per quanto riguasda la successione cronologica degli eventi, si possono distinguere due parti (Boisehot e Pick-Gutmann, 1962; Fokker, 
1963). La prima parte is restituita da un largo spettro continuo, dalle onde centimetriche alle onde nutriche, della durata da 10 mimuti a cirea 2 ore a seconda della intensita del femomeno. Essal ha spesso un inizio piuttosto lappido ed è di frequente associata con bursts di tipo II; in tali casi la radiazione di tipo If di frequenza inferiore ai 100 .HIIz inizia dopo gli outbursts di tipo II, mentre alle frecunenze superiori li precede (Thompson e Maxwell, 1962). Alle frequenze superiori dellit gamma centimetrica, attorno ai $1500 \mathrm{MHz}$, levento procede in modo del tutto sinnle alle diverse frequenze, a creneralmente consiste in un aumento e suceessiva diminuzione di intensita con andimento pinttosto calmo; alle freçuenze inferiori si hammo fluttuazioni di intemsilà più vivaci e più diversificate da frequenze il frequenza.

Talvolta, dopo l'estinzione della radiazione centimetrica a decimetrica, sulle onde metriche l'intensita permane, anehe molto intensa, per alcune ore; "iò costituisce la seconda parte. La distinzione in due parti is giustificata non solo dal fatto che quella che ora abbiamo chiamato seconda parte è costiluita qualsi esclusivamente di onde metrie he, ma anche e soprattutto dalle dire'se (adatteristiche fisiche, come vedremo più avanti, e di direttività: mentre infatti la prima parte ha una rirettività molto bassa, in quanto essa può essere rivelata in eventi she hamno Juego sia al enentro whe a bordi del sole, la seconda presenta

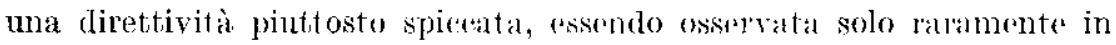
associazione com brillamenti presso il bordo.

Recenti studi (Takakura e Kai, 1961; Takakura, 1962; Kakinuma e Tanaka, 1961; Tanaka e Kakimuma, 1962; Kundu, 1962; Wild, 1962; v. più in generale: Fokker, 1963) hamno dimosteato che uma netta distinzione fisica esiste anche fra le varie tramme di frequenza nella prima parte dollevento; questic ristinzione si manifesta nella posizione delli. solgente, nelte sue dimensioni a nella polarizzaziome della ladiazione emessa. Si noti che quest'ultima catratteristica rivela l'influenza di campi magnetici sull'evento di radioemissione.

La sorgente delle onde centimetriche è in semerale ad un livello pintitosto basso, attonno ai $0,05 R_{0}$ ( $R_{0}$ ragerio dellit fotosfora), ed è di piecole dinmsioni: da $1^{\prime}$ assa temde ad espandersi fino a circa 4'. Il grado di polarizazione si aggira fra il 10 e il $40 \%$; il verso della polarizzazione corrisponde alla propagazione dol lagrgio straordinario, assumendo come polarità del campo magnetico quella della macehia "ovest " del gruppo associato al centro di attività.

Alle onde decimetriche, la sorgente è situata a un livelıo più elevato, raggiungendo $0,3 R_{0}$ al di sopud della fotosfera, e sembra fissa, 0 
quasi, nella sua posizione: le sur dimensioni laggiungono alcuni primi di arco. La polarizzazione è di virso oppesto al quella della radiazione rentimetriea, cioè corrisponde alla propalgazione del ragyio ordinario, $\mathrm{e}$ l'aggiunge il $100 \%$.

Infine, alle onde metriche las sorgente, di dimensioni fra i $6^{\prime}$ a i $12^{\prime}$, si spostat verso l'alto alla velocitu di risca $1000 \mathrm{~km} / \mathrm{sere}$ e si trova nella corona fira 0,5 e $5 R_{0}$ al di sopra della fotosfera. La polarizzazione è in media piuttosto debole, ma soprattutto molto irregolare (Fokker, 1963), ed è difficile stabilire se essisa ror'isponda alla propagazione del raggio ordinario o del ragrio straordinarie.

La radioemissione metrica della sexonda parte dell'outburst di tipo IV appare fisicamente disrinta, come già si è accennato, dalla radiazione metriea della primia parte; lelemento fondamentale della distinzione è rappresentato dalla polarizzazione, molto più intensa nella seconda parte che nella prima. Sulla sorgente e sui snoi moti non si hanno ancora conoscenze sufficienti; ma indubbiamente qualohe diffelenza fisica notevole è assai probabile uhe vi sia, conse è suggerito dalla sensibile differenza nella direttivitì delle due ladiazioni. Boischot e Pick-Gutmann (1962) assumono (he la radioemissione della seconda parte sia praticamente dello stesso tipo di quella delle cosidette "tempeste di radioemissione ", cioè di quella padjazione nella wamma delle onde metrich, di notevole intensita e della durata di parecehie ore o anche di griorni, che non e neecessariamente nssociata ai brillamenti ma solo ai centri di attiviti; la sorgente di tale emissione è piuttosto bassa nella corona $\left(0,2-0,4 R_{o}\right.$ a $\left.169 \mathrm{MHz}\right)$ a ha un dimmetro di solo $3^{\prime}$.

Gli outburst di tijo IV non jossono essere attribuiti a oscillazioni di plasma. Innanzi tutto essi si estendono ad una vasta gamma di frequenze o hanno una durata assai più lumga della manifestazione ottica del brillunento; ora, le oscillazioni di plasma devono essere limitate ad una ristretta banda di frequenze, e devono cesside, per la [5.12], contemporaneamente alla causa eccitatrice, che è plausibile assumere di durata non superiore a quella del brillamento. Inoltre, come si è detto precedentemente, le sorgenti degli outbursts di tipo IV che emetitono anche sulle onde metriche ( $\approx 100 \mathrm{MHz}$ ) raggiungono spesso altezze di alcuni l'nggi solari al di sopra dolla fotosfera, alle quali altezze è molío poco probabile, a causa della relativamente bassa densita detironica, che si possano produrre, secondo la [5.11], oscillazioni di frequenza così elevata.

Per spiegare la radiormissione degli outbursts di tipo IV è quindi necessario fare ricorso, secondo il suguerimento di Boischot e Denisse (1957), alla ipotesi che si tratti di radiazione di sincrotrone, emessa da 
elettroni aventi energia dell'ordine dei $\mathrm{MeV}$ in un eampo magnetico dell'ordine degli oersted. Una particella di carica $f$ e di energia $E \gg m_{0} c^{2}$ in un campo magnetico $H$ perde (a)ergria (si veda ad es. Alfvén e Herlofson, 1950), emettendo onde elettromannetiche, secondo la es. pressione

$$
-d E / \lambda t=\left(2 e^{2} / 3 e\right) \omega_{0}^{2} \alpha^{2}
$$

con $\omega_{0}=e H / m_{0} c$ (girofrequenzal per la massil di quiete), e $\alpha=E / m_{0} c^{2}$. Le onde emesse hanno una frequenza

$$
f=\frac{\omega_{0} \alpha^{2}}{2 \pi}-2,8 \cdot 10^{8} H \alpha^{2}
$$

Blettroni di $5 \mathrm{MeV}$ in un campo nagnetico di 1 oersted danno quindi luogo ad una raliomissione di frequenza di circa $270 \mathrm{MHz}$. Eे evidente che uno spettro di energia molto vasto, quale i certamente quel]o delle particelle interessute dal femomeno deI brillamento, e campi magnetici di valore compreso in un intervallo piuticosto ampio, come esistono nelle regioni di un eentro di attività, possono dar luogro ad uno spettro continno di radionmissione rompreso entro una amplissima gamma di fréquenze.

Tutiavia Boiselot e Pick-Gutmann (1962) e Denisse (1960) ritongono che la seconda parte degli outbursts non si possa spiegare mediante radiazione di sinceotrone; ic da notare rhe tale supposizione si basa sulla ipotesi, da Boischot a Piek-Gutmann ammessi, che la radiatzione della seconda parte sia uguale a quella delle tempeste di radioemissione, come abbiamo accemato dianzi. Secondo tali autori, infatti, elettroni di aleuni HeV allaltezza della sorercete di tale badiom issione, che abbiamo visto essere poou alevatid, furderebbero energia molto rapidamente per collisione a il'regriamento; inolt le anche le caratteristiche di polarizzazione sarebbero a sfavore della ipotesi della radiazione di di simerotrone, poiché questa altima nom dà amissione secondo il riggio ordinario, come avviene invere, secondo Boischot a Pisk-Gntmenn nelat radiocmissione in esame. Denlisse ha aranzato lipotesi che si tratiti di emissione dovutil ad effetto Cerenkov nel plasma.

5.6.1.4. Bursts di tipo $r$. - Si trattat di radioemissione su di una larga banda di frequenza, ma prevalentemente inferiore ai $200 \mathrm{MH} \%$ che accompagna molto spesso i bursts di tipo III; in sostanza il tipo V è un "continuo "associato al tipo IIT così comc il tipo IV è un continuo associato al tipo IT (Wild e coll., 1959: Thompson e Maxwell. 1962). 
Tuttavia il tipo $V$ ha una durata molto minore del tipo IV (pochi minuti) anche se la sua intensità è dello stesso ordine, o poco minore.

Secondo Weiss e Strwurt (1965) la sorgente dei bursts di tipo V è per lo più stazionaria; tuttavia in un certo numero di casi si notano dei moti della sorgente attraverso il disco solare con velocità maggiori di $2000 \mathrm{~km} / \mathrm{sec}$. Generalmente lia sorgente del tipo $\mathrm{V}$ si trova nelle vicinanze di quella del tipo III ad esso associata, a distanza non maggiore di $R_{o} / 3$.

Può darsi che il tipo $T$, come il tipo $I V$, sia dovuto a radiazione di sincrotrone; secondo il modello di Weiss e Stewart (1965) esso potrebbe essere tuttavia prodotto da oscillazioni di plasma gencrate nella corona entro un vasto intervallo di altezze (e quindi in un vasto intervallo di frequenze) da elettroni appartenenti alla stessa nuvola che ha provocato il burst di tipo III, ma che sono stati catturati dai campi magnetici del centro di attivita e cuidati hungo le loro llince di forza fra punti di riflessione (in modo analogo alle particelle nelle (inture di Van Allen).

5.6.1.5. Abbiamo fin qui descritto singolarmente alcuni degli aspetti della radioemissione associata ad un brillamento; agli effetti di una visione completa del fenomeno è tuttavia necessario studiare la coneatenazione degli eventi deseritti e la loro sucessione temporale.

L'andamento tipico della ladioemissione associata ad un brillamento di notevole importanza è all'incirca il seguente. Contemporaneamente all'inizio del brillamento si ha un breve ma intenso fiot to di radioemissione, che alle onde metriche è costituito da un gupupo di bursts di tipo III, seguito da emissione di tipo $\mathrm{V}$; questo prino evento ha una durata di pochi minuti. A questa prima fase segue, spesso dopo una pausa di qualche minuto, un outburst di tipo II, della dulata di aleune decine di minuti, che al termine si confonde con un intenso "continuo" di tipo IV il quale, come già si è detto, può persistere per varie ore.

Questo andamento, che potremmo chiamare "completon, non i tuttavia molto frequente: assai spesso maneano una o più delle fasi descritte. In particolare non è detto che esista la combinazione tipo II tipo IV: vi possono essere outbursts di tipo II senza la emissione di tipo IV e viceversa. La percentuale delle diverse "esclusioni " non ¿̀ ancora ben precisata; i valori dati dai vari autori dipendono evidentemente dal particolare periodo preso in esame. Secondo Maxwell, Thompson e Garmire (1959), fra il primo ottobre 1956 e il 30 settembre 1958 solo sette di sessanta outbursts di tipo II crano seguiti (entro un periodo di 15 minuti) da radioemissione di tipo IV; secondo Kundu 
(1962) nel quadriennio 195i-60, su 6.1 outbursts di tipo IV, 35 elano associati a outbusts di tipo 1I. Questoltimo autore rileva inoltre delle significative dilferenze fra "renti solati, in relazione allat associazione 0 alla mancata associazione tra tipi II e tipi $I V$ : quanto maggriore is l'importanza ottica del brillamento, tanto magurore è la probabilità che esso sia seguito da un tipo IV con tipo II, e altrettanto si puó dire della intensita della radioemissione sulle onde antimetriche associata al tipo IV, nel senso dhe quest'ultima è meno intensa perr i tipi IV senzat tipo II che per quelli associati.

Risultati molto sinili somo stati oftenuti da Thompson e Mraxwell (1962), in seguito alle osservazioni di un quinquennio, dall'ottobre 1956 allottobre 1961: secondo questi antori il 65\%, dexli outbursts di tipo IV e associato ron outhums di tipo TI, a l'85"' con bursts di tipo IIJ.

5.6.2. Radioemissiome a varinzione lenta. - oltre alla moliomissione concomitante ai brillamenti a di ani abbiamo linora parlato, a alle "tempeste di radioemissione" associate con i centri di attività più che con i brillamenti (v. 5.6.1.3.), esiste anche una componente al variazione lenta, osservata specialmente sulle onde decimetriclue. Tale radiazione presenta molto spesso il matteristico periodo di 27 gionin da determinazioni di posizione dumante edissi soluri e mediante metodi

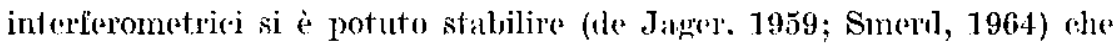
le sorgenti di questa radioemissione corrispondono in generale alle facole di $\mathrm{Ca}$, e sono costituite da regioni a densita superiore al normale ehe si estendono radialnuente a partire dalla fotosfera attraverso la facolo aromosferiehe lino a varie dereine di migliaia li $\mathrm{km}$ al di sopera della fotosfera, vale a dire nella corona. Ia temperat ura equivalente di questa radiazione è dellordine di $10^{6} \mathrm{oK}$; appore quindi probabile da questo fatto, dalla ubicazione delle sorgenti a dalla lontezza della loro evoluzione temporalle, "he si tratti di madiomissione di origine termica, anche se la forma del suo spettro ha potuto destare qualche perplessità (v. Smerd, 1964, p. 80).

\subsection{Protoni solari.}

5.7.1. Polar Cap Absorption (PCA). - In 3.6.4.2 si è accennato al fenomeno dei black-outs polari distinti dai black-outs aurorali, caratterizzati dal fatto che, pur essendo strettamente djpendenti daj brillamenti solari, come le tempeste magnetiche a le aurore, il loro svolgi- 
mento appare praticamente indipendente dallo svolgimento di queste; in particolare, essi hammo inizio generalmente diverse ore prima delle tempeste marnetiche.

Come si è allora detto, si tratta di fenomeni di assorbimento delle radioonde da parte della ionosfera; per la loro cameteristica distribuzione geografica, su cui tomeremo in seguito, si è dato a tale eventi il nome di Polar Cap Absorption, più brevemente indicato dalla sigla PCA.

Il fenomeno consiste nella attenuazione che un'onda elettromagnetica subisce nell'attraversare strati ionosferici dove la frequenza di collisione degli elettroni liberi contro le molecole dei gas atmosferici è rilevante. L'intensità dell'onda è data dallà espressione

$$
I=I_{o} e^{-i k d s}
$$

dove l'integrale è calcolato sul percorso dell'onda. Il coeffieiente di assorbimento $k$ si dedure dalla teoria manetoionica; se la frequenza dell'onla è sufficientemente elevatat da poter considerare trascurabile rispetto ad essa la girofrequenza, ed inoltre è lontana dalla frequenza eritica, si ha per $k$ l'espressione approssimata

$$
k=\frac{2 \pi e^{2}}{m e} \frac{N y}{(1)^{2}} \cdot \frac{y}{y^{2}}
$$

dove $N$ ì la densità clettronian, a la pulsaziono dell'onda incidente, $v$ la frequenza di collisione degli clettroni ad e, $m$ sono rispetiviamente la rarica e la massa dell'elettrone.

Dalle [5.15] e [5.16] si rileva the l'assorbimento espresso in $d B$ è approssimativamente proporzionale al prodotto $N \nu$ : valori anormalmente grandi dell'assorbimento si hamno perciò quando ad una detersminata altezza, cioè con buona approssimazione per una determinata frequenza di collisiona, la densita elettronica cesee al di sopra del suo valore normale a causa di qualche processo di ionizzazione straordinario.

I PCA vengono studiati con vari metodi. Uno di essi è il metodo tradizionale del sondagrio verticale, mediante la misura della minima frequenza riflessa $f_{\mathrm{m} \text { in }}$; la [5.16] mostra infatti che l'assorbimento aumenta al diminuire della frequenza dell'onda incidente; in pratica vengono assorbite completamente tutte le frequenze inferiori ad una frequenza limite $f_{\text {min }}$, funzione creseente del prodotto $N v$, del quale diviene una misura indiretta. Oltre all'inconveniente che il sondaggio verticale è di natura sua intermittente e quindi discontinuo, questo metodo presenta anche quello di ma totale assenza di echi riflessi quando l'assorbimento è molto forte, come av viene spesso durante i PCA, nel qual caso 
esso diviene semplicemente 4 riviatore qualitativo dell'esistenza del fenomeno.

L'inconvenienter ora citato si Iresenenta più raramente nel metodo della "diftusione in avanti " forcard scatto) delle onde ald elevata frequenza (dellordine di parecellie decine di HHz) per tragitti di alcume migliatia di chilometri; tali onde, usate nelle radiocomunicazioni, ineidono obliquamente sulla ionosfera e vengono diffuse e riflesse in aranti da irregolarità locali di densità elettronica, prodotte pen la magrgior parte da seie di meteoriti, cioè in mu intervallo di altezza fra grli 80 e i $100 \mathrm{~km}$. Recentement، Gregory (1962) ha utiliz\%ato la retrodiflusione di onde di media frequenza $(2,3 \mathrm{ML} z)$.

Il metodo the appare migliore is quello del riometro (relatice ionospheric opecity meter). Questo dispositivo è sostanzialmente un ricevitore di rumore cosmico sul frequenzal fissa, generalmente attorno ai $30 \mathrm{MHz}$; lassorbimento viene livelato a misurato dalla allenuaziome del segnale, rhe viene riervuto con continuità. Per misure esatte occorre in realti calcolare il rapponto fra l'intensita del segnale cosmico effettivamente ricevulo a quella del semplale rhe si registra allo stesso tempo siderale in rondizioni ionosferiche alme.

Il riometro $\dot{c}$ un dispositivo che presenta nu certo glato di aselettività ": lalla [5.16] si derluce infatti che, per una determinata densità alettronica $N$, l'assorbimento ì massimo a quelle altezze alle quali la frequenza di collisione $v$ a nugule alla pulsazione 6 dell'onda licevuta. ler un seguale di $30 \mathrm{MH} z$ questa altezza a attorno ai $50 \mathrm{~km}$.

5.7.1.1. Horfologin. - Lat entimuiti di lexpist razione del riometro ha consentito di studiare più a fondo i PCA: si ì constatato che essi si distinguono dai black-out aurorali non solo per la diflerente relazione con l'attività magnetica e aurorale (r. 3.6.4.1 a 3.6.4.2), ma anche per Ie carateristiche della registrayone. I PCA si presentano ron un andamento molto joiù liscio e lento dei black-out aurouali (fig. 5.42). e questo è un elemento dhe permette piuttosto facilmente la individuazione dei due tipi di eventi, a differenza del tradizionale sondaggio verticale. Tuttavia, usando opportuni eriteri suggeriti dalle carateristiche dei PCA emerse grì̀ dai primi casi chiaramente riconosciuti, è possibile utilizzare il gran numero di dati forniti dal sondaggio verticale p«r estendere al massimo il numero di eventi suscrttibili di studio. La deserizione che ora daremo del fenomeno è appunto dedotta dagli studi mediante ionosonde normali di Fakura, Takenoshita e Otsuki (1958), Hakura e Goh (1959), Obaţashi e Makura (1960), Collins, Jelly e Matthews 
(1961), e mediante riometro dia Reid a Collins (1959) e da Reid e Leinbach (1959), montue Baily (1957 + 1959) ha descritto un unico evento (23 f(ubbraio 1956) mediante l'uso dei vari metodi sojula citati, ivi (ompreso) il forward scatter.
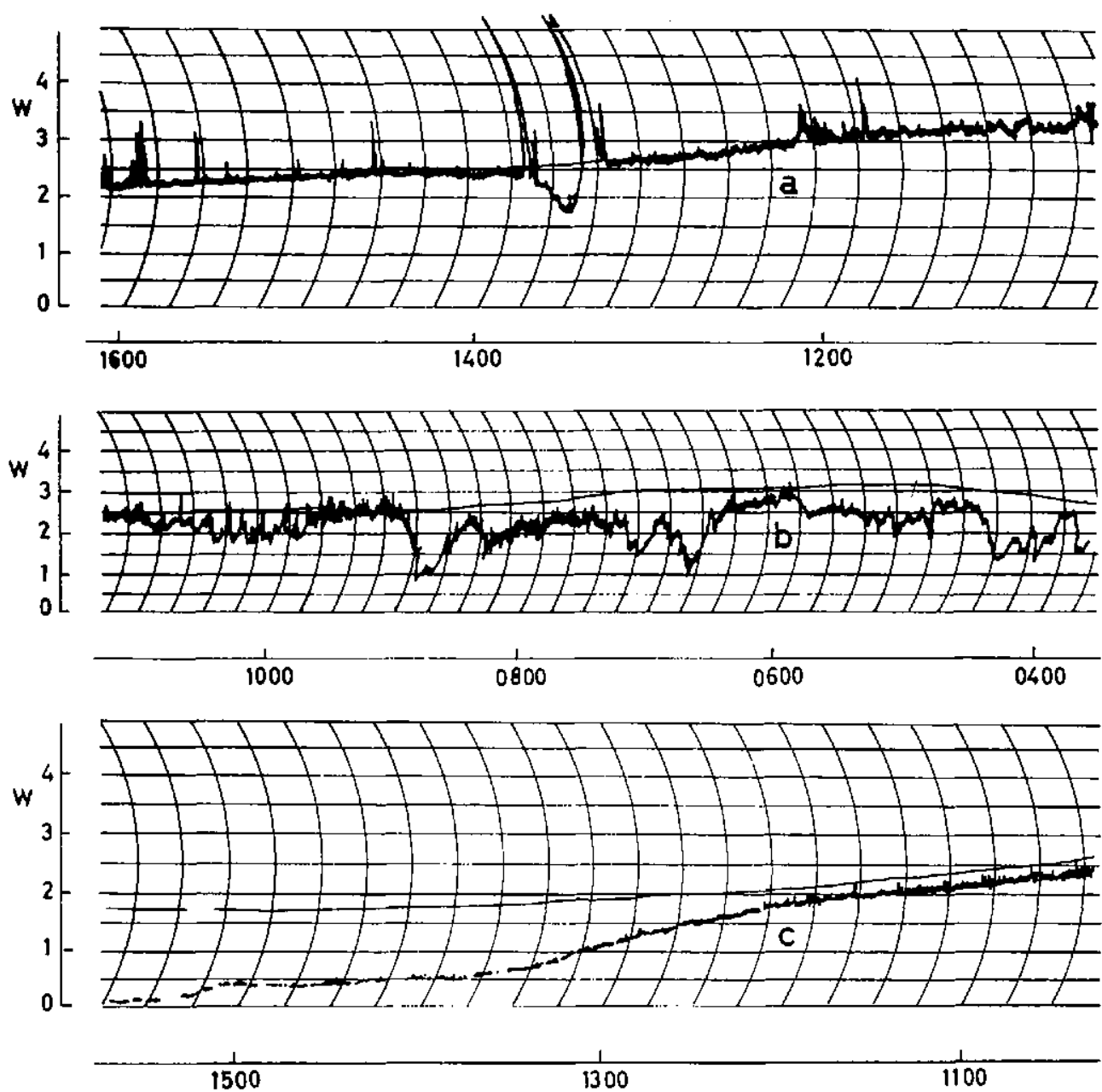

Fig. 5.42 - Esempi di assorbimento anomalo di rumore cosnico: in a) rappresentazione di un SID assoriato con 13 brillamento; in b) di un assorbimento aurorale; in e) di $\mathrm{m}$ PCA. W è la potenza del rumore cosmico isı nuità arbitrarie (secondo Reid e Collins). 
I PCA iniziano in trenerale quitlehe ora dopo il brillamento solare at eui sono associati, con un aumento di assorbinento piuttosto rapido; l'assorbimento raggiunge il massimo dopo alcunte ore, e diminuisce grat dualmente perdurando per qualehe giorno. A questo andanento gasverale si sovappone una netta variazione diurna, con un massimo di assorbimento a mezzogiorno e un minimo a mezzanotte. a con un notevolissimo anmento al sorger del Sole a livelli ionosferiei e di diminuzione al tramonto. Si è già ac'exnnato in 3.6.4.2 alle line'e generali di una spiegazione di tale variazione diurna: la preduzione di ionizzazione, che tende ad ammentare lin densita elettronica $N$, sarebbe continua sia di giomo che di notte; durante le ore notturne però, $N$ diminuirebbe a ransil di una ricombinazione degli elettroni liberi mentre durante il giomo la fotoionizzazione da parte della radiazione solare manterebbe $N$ a valori elevati. Non è noto se della trembinazione siano responsabili le molecole di $\mathrm{O}_{2}$, con formazione di ioni $\mathrm{O}_{2}^{-}$(Bailey, 1959), o se invere si abbia a the fare con un proresso più complicato implieante la formazione di molecele di No (Herzbere, 1960); mal questo problemal non è di interesse inmediato in questa sede.

Iak earatteristien più evidente di questo tipo di assorbimento consiste nella sua distribuzione geografica; rome dice il suo none, esso è limitato alle calotte polari. Secondo i dati csaminati da Babley (1959) e da Stapley a Roberts (1957), dusunte levento suceessivo al grande brillamento del 23 fabbutalo 1956 (il primo P(CA registrato e rjconosciulo) solo Osservatori situati a latitudini geomagnetiche superiori ai $60^{\circ}$ hamo segrlalato un assorbimento notfurno; dai $60^{\circ}$ ai $70^{\circ}$ l'assorbimento anmentava rapidamente di intensità, mentere al di sopra dei $70^{\circ}$ esso rimaneva prationmente costante. In tre aventi prodotti da brillamenti solari nel luglio-agosto 1958 Hultquist (1959) trova che lassorbimento misurato con riometro a Firuna (Iat. greomagn. 65,30 $\mathrm{N}$ ) veniva registrato come black-out anche dalla ionosonda dell'Osservatorio di Tyckself(lat. geomagn. 62,50) ma con durata minore, mentre ad Lppsila (lat. geomatgn. 58, $4^{\circ}$ ) non veniva osservato aleun black-out. Dal canto loro Reid e Leinbach (1959), utilizzando le registrazioni dei piometri in varie stazioni artiche (Alaska), hamno osservato nel periodo masgio 1957-luglio $195924 \mathrm{PCA}$; la durata e la intensità di questi elano pressoché uguali a Thule (lat. geomagn. 89,0 $\mathrm{N}$ ) a a Barrow (latt. geomagn. 68,6 $6^{\circ} \mathrm{N}$ ), ma minori a College $\left(64,5^{\circ} \mathrm{N}\right)$, mentre a Farewell, che $\grave{e}$ a soli $3,3^{\circ}$ geomagnetici a sud di College il fenomeno era assit debole o addirittura mancante. Cio indiea rhe immediatamente a sud di College si trova il limite della zona interessata dai $\mathrm{PCA}$, limite che appare molto netto. 
In generale si può affermale whe i PCA iniziano alle latitudini più "levate, per poi estendersi gladualmente, anche se spesso rapidamente, a tutta la calotta polar fino ai $60^{\circ}$ di latitudine geomagnetica. E importante notare che l'intelnsita dell'assorbimento sembra indipendente dalla longitudine geomagnetirat, almeno nella fase di massimo dell'evento (Bailey, 1959).

All'inizio della tempesta magnetion, whe quasi invariabilmente segue questi eventi, lassorbinento ha praticamente invaso tutta la calot fa polare entro i $60^{\prime}$ di latitudine. Ta perturbazion ${ }^{\wedge}$ magnetica, dalla quale lo svolgimento tempolale del P(A nell'interno di questa calotta è indipendente (come si è gì acesunato), può tuttavia (reare condizioni nuove presso i confini della calotta polare, spostando questi verso latitudini inferiori; anthe Osservatori generalmente immuni da PCA possono allora registrare, sid pure per breve trmpo, un sensibile assorbimento. Secondo Reid e Teinbach (1959), nei 24 "asi sopra citati solo alcune volte l'assorbimento ì stato osservato fino a $7^{\circ}$ a sud di College, $*$ in queste occasioni sempre dopo l'inizio di forti tempeste.

Talvolta invece può accadere il lenomeno inverso: Hultqvist, Aarons e Ortner (1959) registrando a Kiruna mediante riometro l'assorbimento successivo al brillamento del 7 luglio 1958 , assorbimento che poco prima della tempesta magnetica iniziata il giomo 8 aveva raggiunto il valore di $-17 \mathrm{~dB}$, hamno constatato una rapida variazione di esso da -17 a $-5,2$ aB subito dopo l'inizio brusco.

In 3.6.4.2 si è accennato che Hakura e coll. (1958) avevano constatato durante grli eventi anteriori alle intense tempeste magnetiche del 13 settembre 1957 e 11 frobraio 1958 che l'assorbimento aveva luogo contemporaneamente nelle due alotte polari altica e antartica. Chapman (1960) osserva la stessa cosa per il PCA del 7 luglio 1958, notando tuttavia ohe nella regione antartia l'inizio dell'assorbimento sembra ritardato di qualche ol"d lispetto alla regione artica: expli avanza la ipotesi che si tratti di un elitetto dovuto alla spiecata variazione diurna di tale fernomeno, in quanto all'epora dell'evento in esame la calotta polare nord era permunentemente illuminata dal sole, mentre quella sud era in ombra. Jelly e Collins (1962) da uno studio mediante ionosonde in azione nelle regioni polari durante l'Anno Grofisico Internazionale confermano la simultaneità generica dei $\mathrm{POA}$ nei due (rmisferi, ma senza una assoluta contemporaneità nell'inizio, e anch'essi attribuiscono tale circostanza alla carat teristica infuenza della illuminazione solare sui PCA:

$\mathrm{E}$ interessante infine notale un'alt ra raratteristic'd morfologica dei PCA. Basandosi sull'andamento temporale dell'assorbimento nei sin- 
goli eventi, Simno (1961) classifica i PCA in due elassi: i PCA a inizio brusco (tipo S) e i PCA ad inizio graduale (tipo G): la fig. 5.43 rappresenta un esempio di riascuna classe. I PCA del tipo $\mathrm{S}$ ladgriungono il massimo dell'intensità in un tempo relativamente breve (meno di due ore), mentre quelli di tipo (t impiegano diver'se ore. Linteresse di

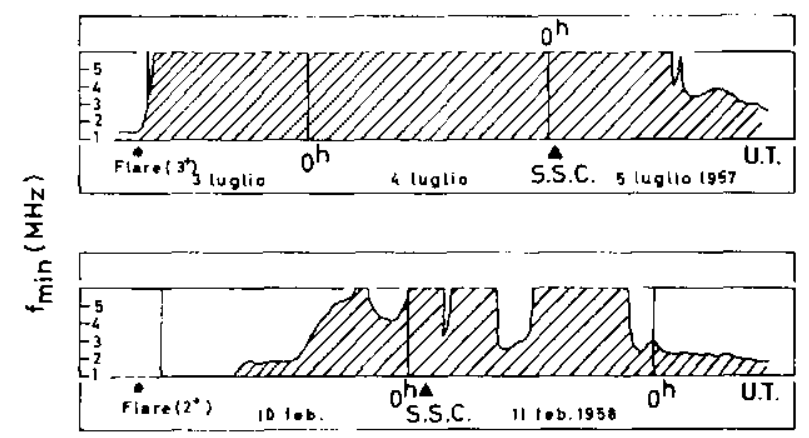

Fig. 5.43 - Esempi di PUA di tipo $s$ (sopra) e di tipo G (sotto) (secondo sinno).

questa classificazione è dovuto, come vedremo, alla particolare correlazione degli eventi delle due classi con la caratteristiche norfologiche dei fenomeni solari the li precedono.

5.7.1.2 Altezza dell'assorbimento. - Già dalla [5.16], che mostra come l'assorbimento sia approssimativamente proporzionale al prodotio $N \nu$, si può arguire che esso sia sensibile ad altezze relativamente poco elevate, dove $y$ assume valori noteroli. I tre metodi di osservazione sopra citati confermano questa conelusione, senza tuttavia poter dare indicazioni molto precise sulle regioni atmosferiche effettivamente interessate all'assorbimento.

Il metodo del sondaggio verticale, eon la misura di $f_{\text {min }}$, mostra semplicemente che l'assorbimento ha luogo ad altezze inferiori alla rerione $E$; in analogia a quanto avviene durante un SID (effetto Dellinger), responsabile del fenomeno è dunque la regione $D$. Il metodo del forward scatter fornisce la stessa indicazione, permettendo inoltre di precisare meglio un limite superiore; il fatto the vengano assorbite onde elettromagnetiche che subiscono la diffusione da parte delle scie dei meteoriti mostra infatti (he l'assorbimento ha luogo ad altezze inferiori alla zona dei meteoriti, ciò̀, come si è già detto, generalmente al di sotto degli $80 \mathrm{~km}$. Il riometro, infine, con le sue proprietà selettive fa riterlere 
possibile, e forsa probabile, the la rexione assorbente si estenda fino ai $50 \mathrm{~km}$.

Conferme a ulteriori precisazioni delle indicazioni precedenti vengono fornite da osservazioni in parte indipendenti dai metodi suecitati. Ad esempio, Landmark (1958), mediante un sondaggrio verticale a impulsi di frequenza fissal $(2,3 \mathrm{MHz})$, ha osservato in Norvegia degli echi da altezze di $65-70 \mathrm{~km}$ durante periodi di forte issorbimento diurno; ciò indiea la pwesenzil di una densità elettronica anormalmente elevata a quei livelli. Inoltre in una esperienza mediante razzi durante un PCA di intensitì moderata Seddon e Jackson (1958) hamno trovato a Churchill una densità eletronica noterolnonte tevata fino ad una altezza di circa $55 \mathrm{~km}$.

5.7.1.3 Relazione con gli eventi solari. - Si ì wià ripetutamente accennato, fin da 3.6.4.2, che i PCA sono strettimente associati a brillamenti solari; gia da allora, quando elano state pubblicate solo le prime ricerche in questo campo, in particolare quella di Hakura e Goh (1959), si profilava una chiara lelazione fra i PCA e i brillamenti arcompagnati da outbursts di tipo IV. Questa relazione è stata nettamente confermatid (Rejd e Leinbach, 1959; 'Thomp)son e Hitxwell, 1960; Kundu e Haddock, 1960; Marda o coll., 1962; Warwick e Haurwitz, 1962; Obayashi, 1962). Sembra ormai ancertato che almeno l'85\% dei PCA è associato con outbursts di tipo IV.

I vari Autori mettono in rilievo, agli effetti di questa assoriazione, la presenza nell'outburst di unil particolare yamma di frequenze: mentre Thompson e Maxwell si riferiscono alle onde metriche, Reid e Leinbarch e in modo speciale Warwick a Iraurwitz atribuiscono notevole importanza alle onde decametriche; a loro volta Kundu e Haddock propendono a ritenere essenziali come cansa dei PCA le onde centimetriche. Date le differenze neggli intelvalli di tempo prese in esame dai vari autori e, talvolta, nella individuazione dell'evento solare causa del PCA, non è ancora possibile decidere con sicurezza se ci sia una gamma di frequenze preferita e quale essa sia; ciò che appare certo è l'associazione di tali fenomeni con eventi soluri di forte intensità.

Incertezze sussistono anche riguardo alla eventuale influenza della posizione dei brillamenti sul disco solare: Reid e Leinbach (1959) a Thompson e Maxwell (1960), basandosi rispettivamente su 18 e 10 casi sicuri, trovano una netta prevalenza di brillamenti nell'emisfero ovest del Sole associati con i PCA. Questa prevalenza appare diminuita nello studio di Warwick e Haurwitz (1962) comprendente 38 casi, e gli autori 
propendono a ritenerlat non signifieativa. Anche Obayashi dallo studio di 62 PCA conclude che non appare unat chiara dipendenza della probabilità di occolrenza dell'evento dalla posizione del brillamento sul sole.

Warwiek e Jlanrwitz e Obayalshi dedicano la loro attenzione anche alla eventualita che dei PCA simo prototli da eventi soliali nell'misfero invisibile del Sole; essi griungono alla colnelusione, notando elue quasi tutti PCA possono venima assortati eon brillamenti eltettivamente osservati, che la probubilità di tasi del genere è assai searsal.

I ritardi fra gli eventi solari e l'inizio de PC. finora osservati famo da cirea 20 minti a circa 50 ore. î interessante corrale se tali ritardi dipendano da particolari caluteristiche di quegli eventi. Sakurai e Mateda (1961) e Marela e coll. (1962) ritengono di poter concludere che esistono due categorie distinte di PCA riguardo al ritarlo di inizio rispetto all'evento solare: i $\mathrm{PCA}$ di "tipo rapido ", aventi un pitardo minore di 5 ore, e quelli di "tjpo lento", con un ritardo maggiore di 5 ore. I PCA rapidi appaiono steptramonte commessi con flussi molto devati di ladioemissione sui $3000 \mathrm{MEz}$ (flussi superiori a 200 unità $\left.10^{-22} W^{-2} H z^{-1}\right)$, mentre quelli lenti sono associati con flussi inferiori alle 100 unita; nessuna relazione appare invere con la intensita della radioemissione sui $200 \mathrm{H} \amalg z$. Anche Kundu e Hatdock (1960) trovano una stretta relazione fra il ritardo dei PCA e l'intensità degli outbursts di tipo IV nella samma sentimetrica: i ritardi minori di 5 ore sono generalmente associati con llussi maygrori di 500 unita sullat intera gamma di lunghezza d'onda di $3.30 \mathrm{~cm}$, mentes con flussi minori i ritardi sono magrgiori di 5 ore. Stecondo Maredal a coll. i dne tipi rli PCA corrispondono ai due tipi morfologici S e G di Simo, alua abiamo

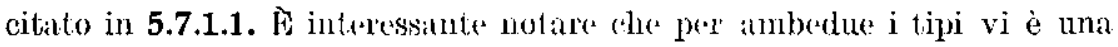
marcata dipendenza del ritado dallat posizione del brillamento sul Sole: il ritardo diminuisce sistematieamente all'aumentane della distanza del brillamento dal borlo est; in altre parole, vi e un notevole effet to Ovest nella distribuzione dei ritardi.

A differenzil della "ellicienza " dei brillamenti nel produre PCA, la dipendenza del ritardo daIIa posizione dell'ponto sul Sole sembra. piuttosto chiara; su questo esiste arcordo fral la maggior parte degli autori. Come fanno notare MeCracken e Palmeira (1960), dai dati di Reid e Leinbaeh si deduce con notevole evidenza una diminuzione del tempo di ritat'do dell'inizio dei PCA dagli eventi solari allo spostarsi della posizione di questi nltimi verso ovest. È significativa la conferma di tale effetto nello studio di Obayashi (1962); la fig. 5.4.t, che riporta $i$ tempi di ritarlo dei POA dall'evento solare e la loro durata, mostra 
con chiarezza l'esistenza dell'effut 10. Inoltre Obayashi nota una differenza anche nel corso sconerale dei $\mathrm{PCA}$ fric quelli prodotti da brillamenti a est e quelli prodotti rla brillamenti a ovest: j primi iniziano più tardi e hanno uno sviluppo più lento, ragrgiungendo un massimo di intensità dopo circa 25 ore dall'oulburst, mentre crli altri, oltre ad iniziare prima,

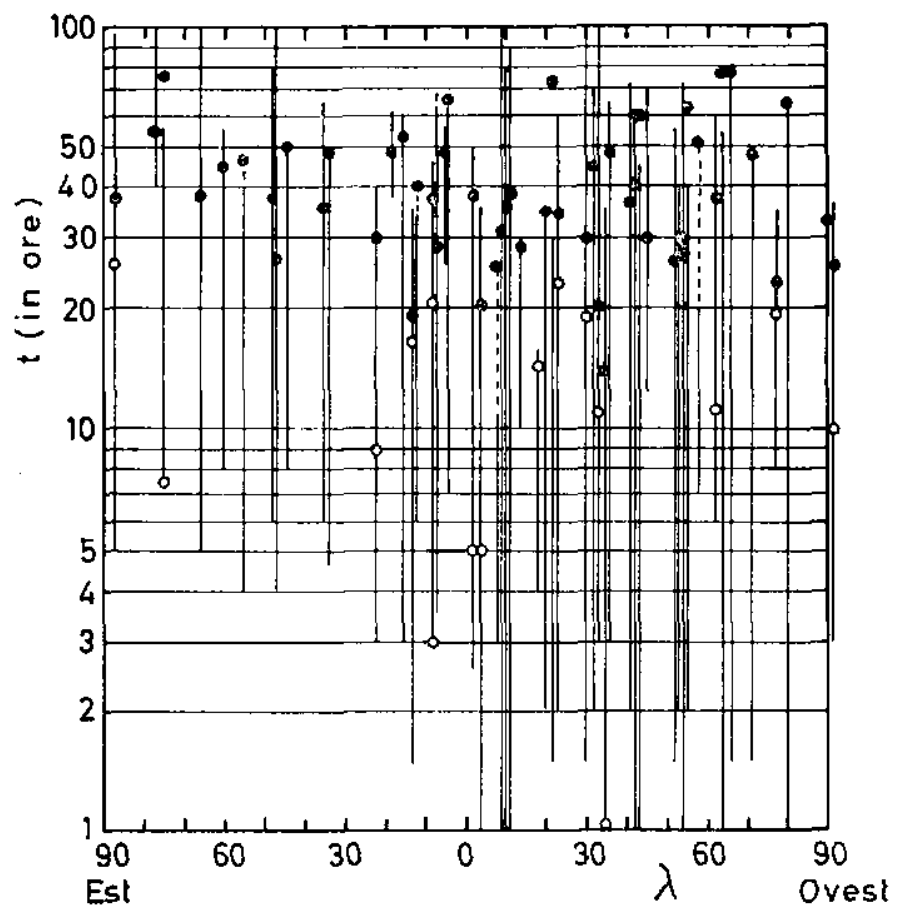

Fig. 5.44 - Dipendenzat del tempo di ritardo $t$ dopo l'outburst dalla longituche eliografica $\lambda$. Le linee continue rappresentano i $\mathrm{PCA}$; eircoletti neri sono s.s.c. di tempeste magnetiche di tipo singolo, i circoletti chiari sono s.s.c. di tempeste di tipo complesso. (secondo Obayashi).

presentano anche umo sviluppo più rapido; dalla fig. 5.45̆ sembra anzi che la differenza nello sviluppo sia anche più cvidente della differenza nel ritardo di inizio.

La fig. 5.45 mette inoltre in luce un'altra distinzione fra i PCA: essi vengono suddivisi dall'Autore nelle due eategorie di "singoli»e "complessi \#; $\mathrm{i}$ "singoli " rappresentuno serie isolate di eventi, costituiti da un solo brillamento e da un solo PCA, con una sola tempesta magnetica associata, mentre $\mathbf{i}$ "complessi " sono costituiti da più eventi 
sovrapposti, dovuti ad un susseguirsi piuttosto rapido di brillamenti di elevatá intensità. $\mathrm{E}$ issai interessante, per quanto si dirà in seguito, la differenza di andamento delle dur entegorir, per cui la seconda $\dot{e}$ caratterizzata da un brusco aumento di intensità dei POA all'istante" di inizio della prima tempesta magnetiea della serje.

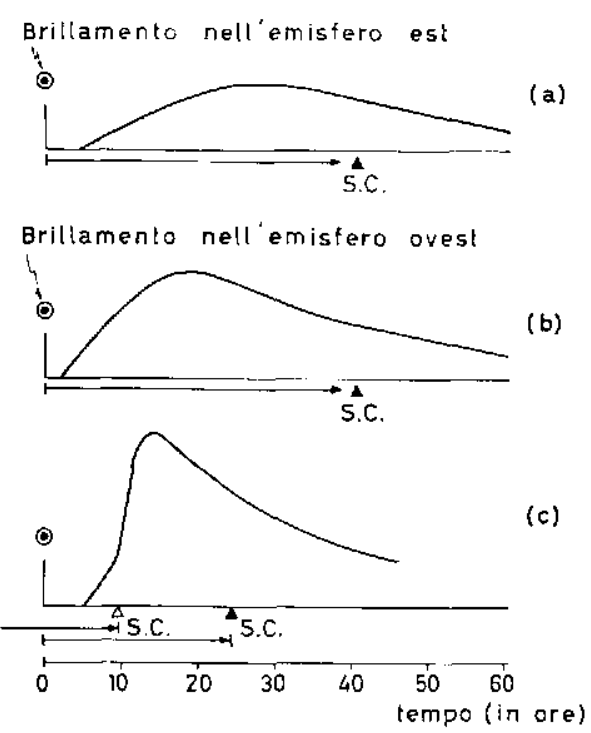

Fig. 5.45 - Variazione temporale media dei PCA singoli (figg. $a, b)$ e complessi (fig. c) (secondo Obayashi).

Tuttavia, Warwick e Hanrwitz mettono in dubbio il suddetto effetto ovest dei ritardi; essi affermano ( $l_{12}$, se mali, i ritardi minori sono associati con brillamenti vicino al moridiano centrale del Sole. Il loro dubbio sembra sostanzialmente basato sul fatto che il cocfliente di correlazione fra lia distanza del brillamento dal bordo est a la velocità apparente dell'ugente produttore dej $\mathrm{PCA}$ è solo 0,23. Di fatto, la fig. 5.44 mette in luce la possibilitì dell'esistenzal dell'effetto ovest senza però che si abbia come conseguenza melevato coefficiente di corralazione fra le due quantità sopra citate, che richiedrebbe, come requisito aggiuntivo alli asimmetria di distribuzione, una relazione di linearità. In ogni caso è un fatto che, come fanno notare Warwick e Haurwitz, sia sufficiente una differenza di interpletazione nell'associazione di un PCA a un brillamento in relativamente pochi rasi per mutare l'impressione offerta dalla fig. 5.44. Gli autori mettono invece in evidenza. che il ritardo sembra dipendente dalla fase del ciclo solare: i ritardi 
diminuiscono dal massimo di attività solare verso il minimo (fig. 5.46). In realtà la differenza fra i valori medi è piuttosto piccola rispetto alla dispersione, tuttavia la sistematicità dell'andamento della fig. 5.46 sem-

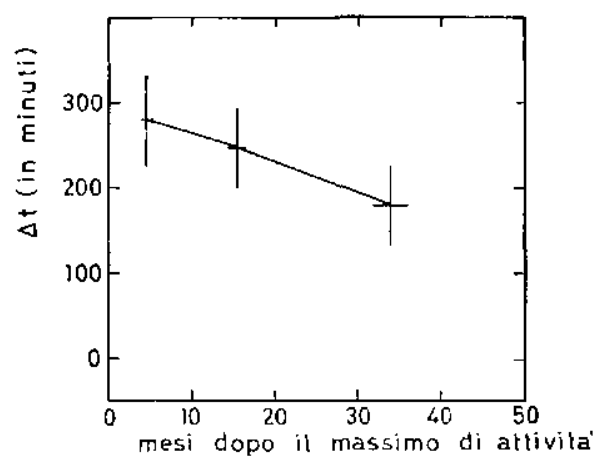

Fin. 5.46 - Andamento del ritardo $\Delta t$ tra brillanento $e$ inizio del $\mathrm{PC} \Lambda$, in funzione della fase del ciclo solare (secondo Warwicli $\theta$ Haurwitz).

bra abbastanza probante; il coefficiente di correlazione fra il tempo dal massimo della attività solare e il ritardo dei PC $A$ è-0,69, la probabilità che tale valore risulti casualmente è solo $10^{-4}$.

5.7.1.4 Natura dell'agente produttore. - Per quanto riguarda la natura dell'agente ionizzante che dà origine ai $\mathrm{PCA}$, si può subito escludere qualsiasi forma di radiazione elettromagnetica. Una prima ragione di tale esclusione è costituita dal ritatrdo nell'inizio dei PCA rispetto all'evento solare di callattere straordinario cui si attribuisce la causa dell'assorbimento; in linea di prineipio si potrebbe anche ammettere che la sorgente della emissione solare responsabile del fenomeno terrestre fosse diversa dal brillamento, ma è bet difheile ginstificare mat tale ipotesi. Un'altra ragione è la particolare dipendenza dei PCA dalla latitudine, dipendenza che non si può spiegare altrimenti che con l'ipotesi di fasci di particelle cariche, controllate quindi dal campo geomagnetico.

Per determinare la natura di queste particelle occorre tencre prosente tre fattori fondumentali: la latitudine geomagnetica a cui avviene il fenomeno, l'altezzal della regione in cui ha luogo la ionizzazione e il ritardo fra evento solare ed inizio del PCA. Questi due ultimi fattori permettono di trarre conclusioni sulla energia delle particelle. 
Un esame di tali elementi sulla base di quanto descritto nei numeri precedenti permette di conclndere con certezza quasi assoluta che non si tratta di elettroni. Infatti, assumendo come tempo di percorrenza

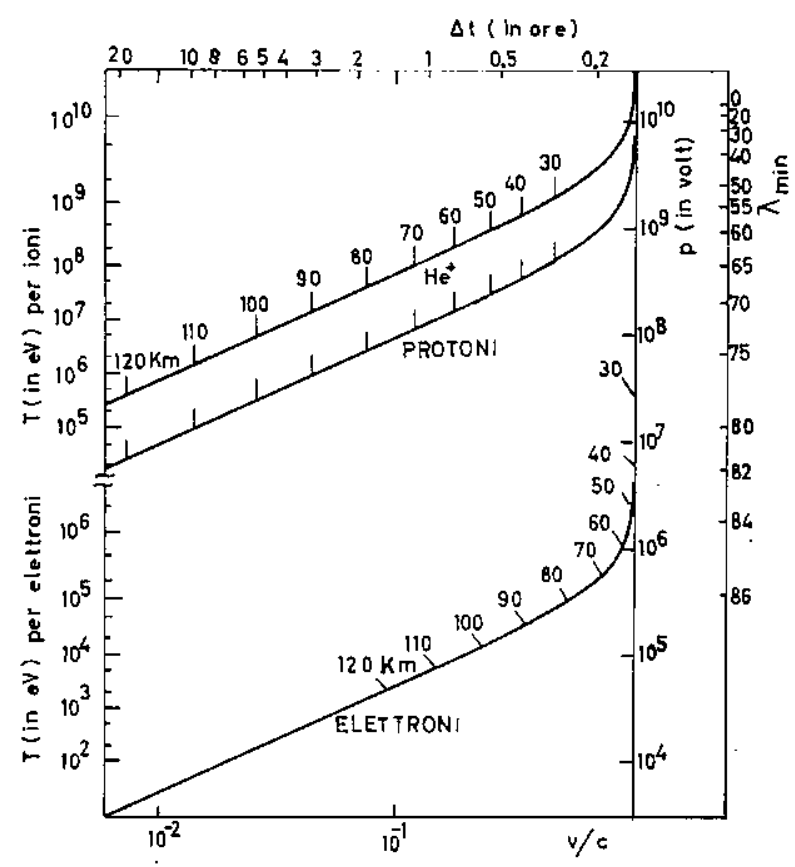

Fig. 5.47 - Altezza di penetrazione per elettroni, protoni e ioni $\mathrm{He}^{+}$in funzione della rigidita magnetica $p$, della velocità $v$ e dell'ener. sgia cinetica $T$. Sulla scala di destra ò riportata la latitudine greomagnetica minima $\lambda_{\min }$ exi possono giungere le particelle di rigiditia $p$; sulla scala in alto tempo di transito $\Delta t$ dal Sole allit Terra su percorso rettilineo (stecondo Bailey).

del tragitto Sole-Terra 30 minuti, che è il temuro minimo finora osservato, e ammettendo ane tale tragito venga percorso in linea retta, si troval che, se le pirticelle fossero elettroni, esse avrobero ura energia di $25 \mathrm{keV}$; dalla fig. 5.47, che riporta il diagramma di Bailey (1959), si deduce che in questo cuso tali elettroni potrebbero giungere solo ad altezze poco inferiori ai $100 \mathrm{knu}$ o a latitulini geomagnetiche non inferiori a $87^{\circ}$. Anche supponendo che il tragitto dal Sole alla Terra non fosse rettiline ma in qualehe modo incurvato e allungato, si avrobbe una insufficiente diminuzione della latitudine di taglio; inoltre l'ipotesi di una deformazione del campo geomagnotico tale da permettere larrivo dexyli elettroni a latitudini inferiori è fuori causa poichè, come si è detto, 
i POA avvengono prima delle perturbazioni magnetiche associate ai brillamenti solari.

Se invece le particelle in questione fossero protoni, per un tempo di percorenza di 30 minuti esse avrebbero una energia di circa $50 \mathrm{MeV}$, giungerebbero fino ar una altezza di poeo più di $40 \mathrm{~km}$ e avrebbero una latitudine di taglio di $65^{\mathrm{a}}-70^{\circ}$; queste sono proprio le condizioni che risultano sperimentalmente dallo stutio dei PCA.

Possiamo quindi concluders che il funomıno dei $\mathrm{PCA}$ è rivelatore della emissione da parte del Sole, durante i brillamenti accompagnati da forti outbursts di tipo IV, di protoni di enerria dell'ordine di 10-100 MeV. Dall'intensità dell'assorłimento ionosferico si può valutare l'ordine di rrandezza del flusso dei protoni incidenti, the ammonterebbe (Reid e Collins, 1959) a cirea $10^{2} \mathrm{~mm}^{-2} \mathrm{~s}^{2} \mathrm{c}^{-1}$, a ${ }^{2}$ duivalente ad una densità del fascio di circa $2 \cdot 10^{-8} \mathrm{~cm}^{-3}$.

5.7.2 Osservazioni dirette di protoni solari. - Le precedent.j deduzioni sulla natura delle particelle causanti i PCA sono state confermate da osservazioni dirette. Di queste osservazioni, eseguite a mezzo di palloni, di razzi e di satelliti in coneomitanza ai $\mathrm{PCA}$, ne esiste orma un numero notevole: sono più di ma quindicina gli eventi di questo tipo rilevati direttamente noll'alta atmosfera e nello spazio circostante alla Terra. La bibliografia su tali osservazioni è assai abbondante, per cui rinuneiamo qui a riportarda pere esteso, rimandando ad alcuni lavori sull'argomento, in particolare a quello di Pieper a coll. (1962) e di Akasofu e coll. (1963) e inoltre alla rissegna di Priley (1964). Coreremo qui invece di rias. sumere le caratteristiche generali della radiazione in esame dedotie da tutte le numerose osservazioni eseguite.

In tutti i casi osservati tale radiazione sembra costituita prevalentemente da protoni; come accuneremo pit avanti, una eerta frazione è formata tutitavia da nuclei più pesanti, in particolare da particelle $\alpha$. Il flusso di partiedle varia notevolmente dir caso a caso: quello finora rivelato dai palloni, a quota dell'ordine dei $30 \mathrm{~km}$, ha oseillato da rirea

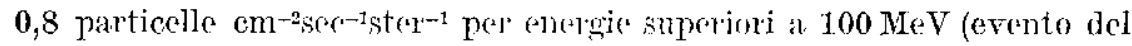
22 agosto 1958: Anderson e coll. 1959), a cirea 240 particelle. $\mathrm{em}^{-2} \mathrm{sec}^{-1}$ ster ${ }^{-1}$ per energie superiori a $88 \mathrm{MeV}$ (evento del 15 luglio 1959: Winckler, Bhavsar e Peterson (1961)). Le misure su satelliti hanno rivelato flussi di vari ordini di grandezza maggiori: il 13 luglio 1961 il satellite Injun 1 ha misurato un flusso di 33000 protoni $\mathrm{em}^{-2} \mathrm{sec}^{-1} \mathrm{ster}^{-1} \mathrm{per}$ energie tra 1 e $15 \mathrm{MoV}$ e un ben scruso flusso di protoni di energia superiore a $40 \mathrm{MeV}$ (il flusso omnidirezionale di questi era di circa $12 \mathrm{~cm}^{-2} \mathrm{sec}^{-1}$ ), 
mentre il 18 luglio suecessivo, di fronte a un fusso unidirezionale di $1000 \mathrm{~cm}^{-2} \mathrm{sec}^{-1} \mathrm{ster}^{-1} \mathrm{per}^{\mathrm{p}}$ protoni fra 1 e $15 \mathrm{~s} \mathrm{ICV}$ veniva rivelato un flusso omnidirezionale di 900 en $^{-2}$ se $0^{-1}$ per protoni di energia superiore a 40 MeV (Pieper e coll., 1962).

Dai valori inclientivi riportati risulta the i flussi osservati sono di vari ordini di grindezza superiori a quello di cir'al 0,1 protoni $\mathrm{cm}^{-2} \mathrm{sec}^{-1}$ ster $^{-1}$ della radiazione cosmica galattica. Per un confronto fra i vari casi occorrereble invee tener conto sia della latitudine di osservazione, sia dello spettro energetico della radiazione incidente.

5.7.2.1 Spettro energetico e di rigidità. - In generale si è ccrcato di rappresentare lo spettro differenziale mediante una espressione della forma

$$
d N=k T^{-\gamma} d T
$$

oppure, ma più raramente,

$$
d N=k^{\prime} p^{-n} d p
$$

dove $T$ e $p$ sono rispettivamente l'energia cinetica e la rigidità magnetica delle particelle.

L'esponente $\gamma$ dello spettro di energia è risultato assai variabile da caso a cho, e, eome vedremo più avanti, variabile col tempo nei singoli eventi. Esso oscilla in generale da 6 a 3 ; nell'evento del 4 maggio 1960 esso ha lappresentato il valore eccezionalmente basso di 1 (Biswas e Freier, 1961). A parte questo valore ecerionilir, to spettro dei protoni solari risulta quindi in gemerale nettamente più ripido sia di quello dei protoni della fiscia interna di Van Allen (v. 5.3.3.1) sia di quello della ladiazioné eosmica galattín (v 4.1.2), cioè è maggione il rapporto fra il numero delle particelle di bassa energia e quelle di energia elevatil.

In generale, dato l'intervallo pinttosto ristretto di energie che in ogni singola osservazinne può essere esaminato, la rapplesentazione secondo spettri di potenza si accorda semple abbastanza bene con le osservazioni. Ma se l'intervallo di enerria esaminato si estende, o se si vogliono accordare per uno stesso avento osservazioni eseguite ad altezze nettamente differenti e quindi interessanti intervalli di energia molto diver'sa (osservazioni con palloni e osservazioni con satelliti), l'uso dello spettro di potenza richiede che l'esponente $\gamma(0 n)$ sia una funzione dell'energia (o della rigidità); in generale $y$ a $n$ diminuiscono al diminuire dell'energia e della rigidità. 
Per studiare più a fondo la forma dello spettro, Freier e Webber (1963) hanno esaminato sei eventi importanti di protoni solari, ricavando i dati sperimentali dalle caratteristiche dei corrispondenti PCA dedotte mediante riometri per energie fra 1 e $50 \mathrm{MeV}$, dai risultati delle osservazioni mediante palloni per l'intervallo $70-500 \mathrm{McV}$, e dalle registrazioni dei neutroni al suolo per energie superiori a $500 \mathrm{MeV}$. Secondo gli autori lo spettro integrale dri protoni solari è ruppresentabile assai bene, piuttosto che come spettro di potenza, eome spettro esponenziale di rigidlità della forma

$$
N=N_{o} e^{-\frac{p}{p_{o}}}
$$

dove $p_{o}$ è una effettiva costante (e non più una funzione di $p$ ), che determina la ripidità dello spettro.

Sotto questa forma, la variabile ripirlità rexli spetiri da caso a caso viene manifestata da una notevolo variabilità di $p_{o}$; nei sei casi esaminati da Freier e Webber $p_{o}$ oscilla tra il valore di $60 \mathrm{MV}$ (spettro molto ripido) osservato il 12 luglio 1961 e il valore di $375 \mathrm{AV}$ (spettro molto piatto) del 15 novembre 1960 .

5.7.2.2 Variazioni temporali. - Una importante caratteristica generale dell'andamento temporale: di un evenlo di protoni solari (che d'ora in poi indicheremo con SPE, Solar Protons Event), è la sua durata, che risulta sempre molto più lunga di qualsiasi manifestazione osservabile dell'evento solare ritenuto come origine. Ciio del resto era stato messo in luce dallo studio dei PCA. L'esempio piu notevole di evento đi lunga durata è quello registrato dit Anderson a Enemark (1960) nel luglio 1959 mediante lanei di palloni a Resolute Bar, a $150 \mathrm{~km}$ dal polo magnetico nord (inelinazione misnetiea di 90\%). Nel campo di energia esplorato (fra 85 ( $^{2} 400 \mathrm{M}+\mathrm{V}$ ), il flusso di protoni associato al brillamento di importinza $3+$ del 16 luglio era ancora registrabile il giorno 27; si noti perr di più che, secondo una interpretazione di Batity (1964) dei PCA verificatisi in quel periodo di forte attivita solare caratterizzato da tre intensi brillamenti il 10, 14 e 16 Inglio, i protoni registrati da Anderson e Enemark dovevano considerarsi associati al brillamento del 10 .

La lunga durata degli eventi indica che la propagazione dei protoni fra il Sole e la Terra è un fenomeno assai complesso, la cui caratteristica più importante è la aecumulazione delle particelle in qualche regione dello spazio extraterrestre; ciò è evidentemente connesso con lo stato 
fisico dello spazio intarplanetario. Di conseguenza is ntile. atgli effrenti delle conoscenza di tali condizioni flstute, studiale il decadimento temporale del flusso protonies incidente sulla Terra ossia lo strotimento delle regioni ali accumulazione.

Risultati su questo punto si hamno, in misue dirote di protomi solari, per gli eventij del luglio 1959 (Andrson * Enemark, 1960), dell'aprile 1960 (Arnoldy a coll. 1960) e del 4 masgio 1960 (Winckler, Hatsley e May, 1961). Per l'evento del heglio 1959 Andelsoll e Enemark trovano un decadimento temporale dell'intensita frotonica fl'al 85 a $400 \mathrm{M} \times \mathrm{Y}$ che si accorda assai bene con la legege $t^{3}$, contando i tempi a partine da 16 ore dopo il brillamento del 16 lus lin. Nellaprile 1960 si sono

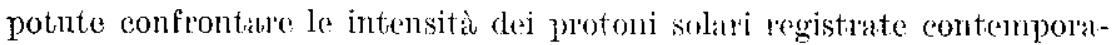

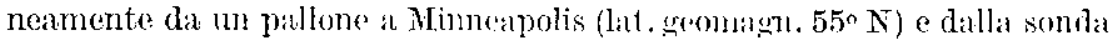
Pioneer V a 5.000.000 kin chatla Terra. In ambedue $i$ casi il decadimento

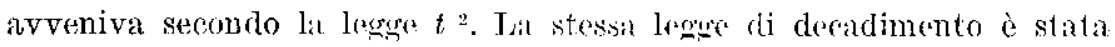
dedotia da Wincliler, Masley a Moy pee lerento del 1 maggio 1960 ,

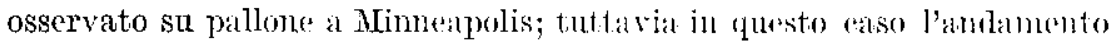
poteva anche essere rappresentato nella formis $t^{-1, b}$.

In realta la rapplesentazione del deeadinento temporale dei protoni secondo und legge del tipo ora citato ha senso solo se essit viene riferita a protoni di una deerminata enereia, o, in altre parole, ad una

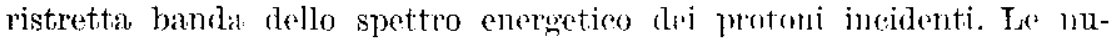
merose misure effettunte hanno infatti portato a concludere che in wa-

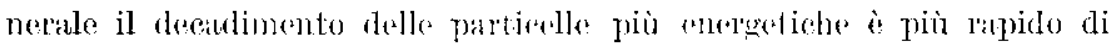
quelle di energia minore, ossia lo spestro energetien dei protoni cliventa col tempo piu ripido. Fra sli SPE importanti, solo quello del 10-12

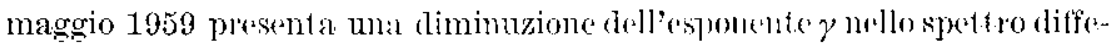

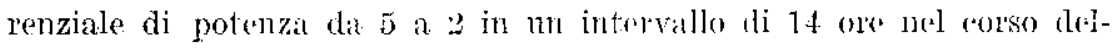
l'evento (Winckler' a Bharsal' 1960); ma tale ablimento è condizionato, rome si vedrà, da altere ratse ar a sovlappongono al semplice decadimento de flusso dei protomi prorenienti disllo spazio.

Particolammente indicativi sull'andamento temponale del flusso di protoni in funzione dell'energia fra $2 \mathrm{MPV}$ " $600 \mathrm{MLV}$ sono i risultati ottenuti dal satellite Explorer 12 nel settembre 1961 (Bryant e coll, 1962).

In una serie di osservazioni a pochi minuti di distanza l'uma dall'altra, a partire da cirea 25 minuti dopo il massimo del brillimento di importanzı 3 del 28 settembre, è stato notato un rapido aumento del flusso di protoni; ma mentre inizialmente i protoni di energia attorno ai $600 \mathrm{MeV}$ presentavaro una intensità maggeriore di quella dei protoni meno energetici, nel tempo di un'ol'a il flusso di questi ultimi 
era diventato nettamente superiore. Is sucessive osservazioni, eseguite ad intervalli di trmpo magriori, hamno chiaramente mostrato l'inizio del decadimento dei protoni di energia superiore ai $1.00 \mathrm{MeV}$ quando il flusso di quelli di 3-10 $\mathrm{H} \cdot \mathrm{V}$ ara ancora in aumento; tale allmento si e protbatto fin verso la fine del giomo 30. Rappresentando lo spettro energetico ron lma aspressione di polen\%a, si ottiene nell'intervallo da 3 a 600 Afor per lo spettoro differemziale $\gamma=2$ il giomo $29 \mathrm{e}$ $\gamma=3$ il griorno 30 .

Si noti che la novita nelle osservazioni con satelliti rispetto a quelle con palloni è costituita latlat possibilità di otliturere informazioni sulle energie molto basse, al di sotto dei $10 \mathrm{MeV}$.

Se si confrontano i risultati sull'andamentio temporale del flusso protonico durante gli SLE ottenuti mediante sialelliti e mediante palloni a latitudini diverse, si notano delle evirtenti differenze. I lanei di palloni a latitudini molto clevate, quale quella di Ft. Ohurehill (lat. geomagn. $68,7^{\circ} \mathrm{N}$ ) hanno rivelato l'arrivo di protoni poco tempo dopo il brillaunento solare inssoriato all'rverito: escimpio tipico è quello del 22 agosto 1958 (Anderson : roll., 1959), in eui il flusso di protoni si è fatto notevole cires un'ora dopo il brillamento, praticamente in coincidenza con

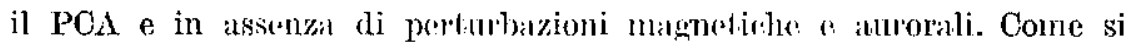
deduce dalle osservationi di Anderson e bnemark (1960) per uno degli eventi del luglio 1959, osservizioni affelluatis con palloni laneiati a Resolute Bay, il deceulimento del flusso dopo Jintensit a massima avviene assai regolanemente; in maniern del tatto indipendente dalla tempesta magnetion sucessiva al brillamento. D'altra parte nei lanei effettuati a Minneapolis (Winckler, 1960; Winckler, Bhawsar e Peterson, 1961) intensi flussi di protoni solari si sono osservati solo durante la fase prineipale delle tempeste mutenetiche; lum fatifo assai interessarnte in questi casi è che venivatno rivelati protoni di enerria dell'ordine di $90 \mathrm{MeV}$, cioè ben al di sotto della normale eneruia di agrlo per Minneapolis, di circa $350 \mathrm{MeV}$. Risulta quindi evidente che durmte la perturbazione magnetica l'andamento delle linee di forza attorno alla Terra viene deformato in misura assiti sensibile; ciò del resto è in accordo con quanto già trovato per i PCA (v. 5.7.1.1), i quali si estendono verso le basse latitudini al momento della tempesta magnetica associata all'evento solare che ha dato orimine al PCA stesso.

Alla luce di questá constatazione si può spiegare la diminuzione dell'ssponente nello spetiro di potenzal dei protoni nell'evento del 12 maggio 1959 osservato con palloni da Wincklel e Rharsar (1960). L'osservazione veniva effettuata a Minneapolis, dove i protoni risultarono pre. 
senti solo durante la tempesta magnetiea; rjperendendo il campo magnetico gradualmonte verso la fine della tempestal la sua confinumazione normale e riorescendo le energie di taglio, a si attende enfettivamente che il flusso di protoni di energia minore diminuisea pin rilpidinnente che per quelli di ensergia più elevata, riducendo con cio liı ripidità dello spettro.

In realtà il taglio greomatgretico pex protonj solari è risultato anche in condizioni masneticamente calme minore di quello galcolito sia in base alla teoria di Stömer per un sampo di dipolo sia in base ad una migliore approssimazione del eampo geomagnetico; ad esempio l'ieper e coll. (1962) mediante strumenti a bordo del satellite Injun I osservamo protoni da 1 a $15 \mathrm{MeV} 18$ ore jurima dell'inizio brusco della tempestat magnetiea del 13 luglio 1961 it una latitudine geomagnetion di 630, cleve secondo la teoria di störmar potrebbero giumgere solo protoni di enerengia superiore a cires $200 \mathrm{MeV}$.

Le osservazioni con satelliti hamno pivelate un'altra differenza nel-

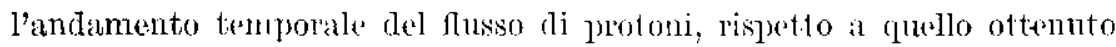
con i palloni ad elevate latiturlini. Si è poeo faz ricordata l'osservazione di Anderson e Enemark (1960) sulla indipendenza dalle perturbazioni magnetiohe dell'intensità dej protoni misututi con palloni a Presolute Bay; tale risultato vale pre la banda di enceluta osservabile, cioe fra 85 e $400 \mathrm{MeV}$. Nol manpo delle thergit minori (dat 1 a $15 \mathrm{MeV}$ ), che $\mathrm{i}$ satelliti possono rivelare, tale indipendenza non sussiste più. Il satcellite Injum I ha registrato durante la tase iniziale della tempesta magnetica del 13 luglio 1961 un fortissimo aumento mel flusso dei protoni entro quell'intervallo di conergia; un rapido decadimento dell'intensità ha avoto luogo allineirea dumente la trasizione dalla fase iniziale alla fase principale dalla tempesta. That diminuzione analogat, and se so nel romplesso l'intensità del fenomeno era minore', è stata osse! lauta frat lat fase iniziale e quella principale della tempesta del 20 luglio. Anche il satellite Explorer XII ha registerto un forte aumento dolla intensita dei protoni di encergia inferiore a $15 \mathrm{Mr} \times \mathrm{V}$ in compispondenza all'inizio della ternpesta del 30 settembre 196.1. Sula interpretazione di tali risultati torneremo in seguito in $\mathbf{5 . 8}$.

Un altro aspetto importante delle variazioni temporali dei protoni solari è costituito della isotropia delle particelle incidenti sulla Terra. Già descrivendo la morfologia dei PCA abbiamo visto che essi si distribuiscono eon grande uniformità su tutta la alotta polare, con una intensita che appare indipenderte dalla longitudine geomagnetica; cio è un chiaro indice della isotropia delle direzioni di incidenza delle parti- 
anle. Ha lo ossetrazioni di Explorer XII hamno fornito informazioni dirette sulla formazione della isotropia durante l'evento del 28 settembre 1961. Xell'intervallo di energie fra 100 e 600 MroV nelle primissime fasi dello SPE le particelle presentavano ma mateatar anisotropia, che ò andata gradualmonte diminnento per seompario completamente dopo circa mora e mezzal dal brillamento, prima dhe l'intensità del flusso raguriungesse il sno massimo. Tol rampo delle basse encergie, fra 2 e $15 \mathrm{MeV}, \mathrm{l}$ anisotropia si a potrith per cirra setfe ore dopo il brillamento, e, a differenza dei protoni più velori, anclè dopo aves raggriunto il massimo di intersita. Le direzioni preforenziali di provenienza delle particelle nei due intervalli di enereia non apparivano coincidenti.

5.7.3 Particelle a e nuelei piu pesanti. - Por una decina di eventi sono stati studiati intersità e spettoo di eventuali nuclei diversi dai protoni presenti insione a questi nltimi (Firlted a Guss, 1962; Biswas, Fichtel e Guss, 1962; Biswas, Freirer a Stcin, 1962; Ney e Stein, 1962; Biswas, 1962; Freier, 1962 a 1963; Biwwas e coll., 1963; Freier e Webber, 1963). Tale stadio è stato eseguito mediante emulsioni nucileari lanciate con palloni e con razzi e suecessivamente recuperate.

I dati sperimentali raccolti non sono in realta molto numerosi e non permettono aneora una descrizione sistemitica dolle earatteristiche dei nuclei pesanti duranf wli SPE. Tutravià è accertata la presenza durante molti di questi eventi di particelle $a$, spesso con flussi notevoli.

Il rapporto misurato fra l'intensita dei protoni a quella delle particelle a varia evidentemente a seconda del parametro the si seeglie come riferimento $p^{3} \mathrm{r}$ il confronto: si possono infatti paragonare i flussi di protoni e particelle a nello stesso intervallo di ancroia per nucleone (il ehe equivale allo stesso inteprallo di velocidi). oppure nello stesso intervallo di rigiditid. E) tuttavia sperimentalmente stabilito che il rapporto protoni/particelle $\alpha$ è estuemamente variabile da evento ad evento; mentre in qualche evento (come in quelli del nov. 1960) l'intensità delle? particelle $a$ la presentato, almeno por certi intervalli di tempo, valori praticamente uguali a quelli della intensità protonica, in altri assa scende a qualehe unità per cento. Vi sono inoltre indicazioni di un decadimento col tempo più rapido per le particelle $a$ che per i protoni. Secondo Freier e Webber (1963) anche le particelle a presentamo, come i protoni, uno spettro esponenziale di rigidita avente la stessil costante po dello spettro dis protoni.

In qualehe evento (luglio 1959 , settembre 1960, novembre 1960) sono stati studiati ancele i nuclei di numero atomico maggiore, rag- 


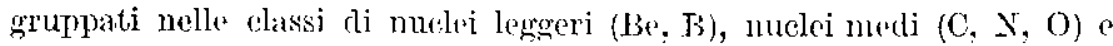
nuclei pesanti $(\%>10)$. Il rapporto fra l'intensiti dei nuclei medi e quella dei protoni is in scenerale dell'oreline di 10-3. Secondo Firhtel a Guss (1962) e Biswas, Fiehtel e Guss (1962) dhe hammo sturliato arematamente gli erenti del novembre 1960, Jia composizione dej fisce corpuscolari, ossia il rapporto fra le intensiti dei vari tipi di nuclei, è in buon aecordo con la composizione dell'atmosfera solare, ma significativamente diversa dalla composizione dei raggi cosmici galattici.

\section{BIBLIOGRALIA}

A thabaxli K., Collen M. H., Astroph. Journ. 133, 258 (1961).

AKasofu S. I., LiN W. O., Vax Aldex J. A., Jourmal. Geoph. Res. 68, 5327 (1963).

Alfokn 11., T'ellus, 8, l (1956).

Alfyen 1I., Ifertomson N., Phys. Rev. 78, 016 (1950).

Alfón H., Lehinerí B., Yalure 178, 1339 (1956).

ALLEN C. W.. Month. Not. Zoy. Astr. Soc. 107, 426 (1947).

Anderson K. A., drinoluy R., Homman R., Petersox J., Winckier J. R., Joum. Geoph. Res. 64, 1133 (1959).

ANDerson K. A., Exmank 1). C., foum. Geoph. Fers. 65, 2657 (1960).

Arvotdr R. [., ILorimax R. A., Winchete J. R., Joum. Geoph. Res. 65, $3004(19060)$.

Babcock H. W., Astroph. Journ. 118, 387 (1953).

B авсоCK H. D., Astroph. fourn. 130, 364 (1059).

Вавсоск Н. W., Вавсоск Н. J., Astroph. Journ. 121, 349 (1955).

BaILEY T. K., Journ. Gicoph. Res. 62, 431 (1957).

BaILET D. K., PIHE 47, 255 (1959).

BaIfey D. K., Plan. and space seience 12, 495 (1904).

Bitonsle R. V., MCNinRy I. R,, Astroph. Journ. 139, 1312 (1964).

BISWAS S., Joum. Geoph. Lies. 67, 21313 (1962).

Biswas S., Fion'ted (1. E., Guss D). li., lhys. Rev. 128, 2756 (1962).

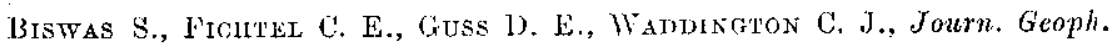
Res. 68, 3109 (1963).

Biswas S., Freive 13. S., Journ. Geoph. Res. 66, 1029 (1961).

Biswas S., Freier 1'. S., Stein W., Journ. Geoph. Res. 67, 13 (1962).

Borscro't A., O. K. Acad. Sci. T'uris 244, 1326 (1957).

Borschot A., Denissi J. F., C. R. Acad. Sci. Paris 245, 2149 (1957).

Borschot A., Pron-Gutman M., Jaum. Phys. Soc. Japan 17, Suppl. A-II, 203 (1962). 
Broxisox J. W., Ihys. Fev. 62, 521 (1942).

Bryant D. A., Crine 'l' L., Desar U. D., McDoxald F. B., Journ. Geoph. Res. 67, 4983 (1962).

Cifapdax J. H., Ganad. Journ. Phys. 38, 1195 (1960).

Cones H. H., Astroph. Journ. 130, 221 (1959).

Confes M. H., Astroph. Journ. 133, 978 (1961).

Collins C., Jelly D. H., Matrirews A. G., Canad. Journ. Phys. 39, 35 (1961).

Correl M., Jazlex M., Baing J., Astroph. Joum. 124, 597 (1956).

Correl M., Roberte W. O., Astroph. Journ. 127, 726 (1958).

Contrez R., Radioastronomia Solare, Scuola Internazionale di Fisica "Enrico Fermi ", Corso, XI I, p. 207 (1960).

Covbive T. G., Wonth. Yot. Roy. Astr. Soc. 106, 218 (1946).

Denisse J. F., E.R.S.I. General Assembly London, (1960).

Frchtel C. E., Grss D. E., Journ. P'hys. Soc. Japan 17, Suppl. A.II, 321 (1962).

Fokklin A. D., Space Science Rev. 2, 70 (1963).

Freier P. S., Jounn. Geoph. Res. 67, 2617 (1962).

Friter P. S., Journ. Geoph. Res. 68, 1805 (1963).

Freier P. s., Webrer W. R., Jourm. Geoph. Res. 68, I605 (1963).

Gopala Rao V. U., Austral. Joum. Phys. 18, 283 (1965).

Gopasyue S., Ogre M., severiy A., Simposinikova E., Publ, Orim. Astroph. Obs. 29, 15 (1963).

Gregory J. I3., Journ. Geoph. Res. 67, 3829 (1962).

II akura Y., Goir 'T., Journ. Radio Res. Lab. Japan 6, 635 (1959).

HakURa Y., Takexoshípa Y., Otsuki T., Rep. Ion. Res. Japan 12, 459 (1958).

HALE G. E., Astroph. Journ. 28, 1000315 (1908).

IIALE G. E., Astroph. Journ. 38, 27 (1913).

Hale G. J., Nichorson \$. B., Papers Mt. Wilson Obs. 5 (I, II) (1938).

Hale G. E., Shares F. II, v. MaANen A., Eldermay F., Astroph. Journ. 47,206 (1918).

Herzberg I., Journ. Geoph. Res. 65, 3505 (1960).

Hoctgast J., van Sluiters A., Bull. Astron. Inst. Nelherl. 10, 325 (1948).

How ARD R., Astroph. Journ. 130, 193 (1959).

Howard R., Slellar and Solar Magnetic Fields, I.A.U. Symp. 22, 129 (1965).

How ARD R., BABCoCK H. W., Astroph. Journ. 132, 218 (1960).

Howard R., Severxy A. B., Astroph. Joum. 137, 1242 (1963).

Hughes M. P., IInkxess R. L., Astroph. Journ. 138, 239 (1963).

Hultqvist B., Tellus 11, 332 (1959).

IIUtctqust B., Anrons J., Ortnek J., Tellus, 11, 319 (1959). 
Jager C. de, fineyel. of Phys. 52, 296 (1959).

Jager C. de, ladioastronomia Solare. Scholit Internazionale di Fisica "Enrico Fermi "Corso XII, p. 34" (1960).

Jelty D. H., Coteriss O., Ganad. Joum, thys. 40,706 (1962).

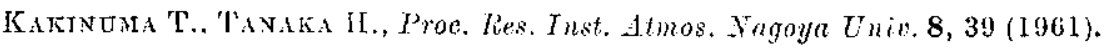
Komssarolit W., Austral. Joum. Phys. 11, 33s (1958).

Kundu A. R., Joum. l'hys. Soc. Japan 17, Supht. A-II, $21 \overline{5}$ (1962).

Kundu M. R., HADDock F. 'L., Nature, 186, 610 (1960).

IJANDMARK B., Journ. Atm. Terr. Phys. 12, 7!) (1958).

Inightox R. B., Astroph. Journ. 130, 360 (1959).

Jatiriton R. B., Stellar and Solar Magnetic Ficlds, I.A.U. Symp. 22, $158(1965)$.

Maeda ll., Sajurat K., Oxnon 'T., Yamamoto M., Ann. Géophysique 18, $305(1962)$.

Mat'TiG W., Zeitschr. Astroph. 31, 273 (1953).

Matтr, W., Zeitschn. Astroph. 44, 280 (1958).

Maxwele A., Swarep G., Nature, 181, 36 (1958).

Maxtell A., 'Jinmpson A. R., Astroph. Jounn, 135, 138 (1962).

Maxwhl. A., 'I'tompson A. R., Garmire G., I'lan. and space Science 1, $325(1959)$.

McGracken K. (x., Palmina R. A. R., Joum. Geoph. Res. 65, 2673 (1960).

NewKtRK G., Astroph. Journ. 133, 983 (1961).

Ney L. P., Stein W. A., Journ. Geoph. Res. 67, 2087 (1962).

Nicholson S. B., P'ubl. Lstronom. Soe. Pacifie 45, 51 (1933).

Obayasifi 'T., Journ, Geoph. Res. 67, 1717 (1962).

Obayashi 'T., Haklia Y., Rej. Ton. Space Res. Japan 14, l (1960).

PAWSEY J. I., SMERD) b. H., in the Sun, edit. Kiniper, The University Press, Cliciogo, 47t (1953).

Piephis G. F., Zmuda A. J., Bostrom C. O., O'Brien B. J., Joum. Geoph. Res. 67, 4959 (1962).

REID (r. C., Corlins C., Journ. Atm. Terr. Phys. 14, 63 (1959).

ReId G. C., Lemsach II., Journ. Geoph. Res. 64, 1801 (I959).

Richatrdson R. S., Astroph. Joum. 107, 7 (194s).

Roberts J. A., Austral. Journ. Phys. 12, 327 ([959).

SARURAI K., MAlid H., Journ. (feoph. Res, 66. 1966 (1961).

SEddon J. C., JACKSON J. E., IGY Rocket Reporl Series 1, 140 (1958).

Seares F. H., Astroph. Journ. 38, 99 (1913).

Severny A. B., Publ. Crim. Astroph. Obs. 31, 126 (1964).

Styeriy A. B., Stellar and Solar Magnetic Fields, I.A.U. Symp. 22, 141 (1965a).

Severny A. B., Stellar and Solar Magnetic Fields, I.A.U. Symp. 22, 238 (1965b). 
Severny A. B., Stellar and Solar Magnetic Fields, I.A.U. Symp. 22, 358 (1965)

Shapley A. II.. Roberts W. (J.. Nat. Bureau of Slandards, Report 5062 (1957).

Snmon G. W., Thesis, California Institute of Technology (1963).

Sinno K., Journ. Radio Res. Iab. Japan 8, 17 (1961).

SHE S. F., Solar Radio Emission, in Research in Geophysics (edit. IIugh Odishaw), 1, 65.97 (1964).

Suerd S. F., Westroto K. C., Phil. Mag. 40, 831 (1949).

stenflo J. O., Obsernalory (in corso di stampa) (1966).

Strpanov V., Seversy A. B., Publ. Crim. Astroph. Obs. 28, 166 (1962).

Steware'1 R. 'l', A ustral. Joum. Phys. 18, 67 (1965).

Thkakuka T., Joum. Pliys. Soc. Japan 17, Suppl. A.JI, 243 (1962).

Takakura T., Space sici. Rev. 5, 80 (1966).

TAKakURa T., Ka K., Publ. Aslr. Soc. Japan 13, 94 (1961).

'Tanaka H., Kakixuma 'l', Journ. Phys. Soc. Japan 17, Suppl. A-II, 211 (1962).

Thressex G., Naturwiss. 40, 218 (1953).

'Jimompson A. R., Maxwei. A., Nature 185, 89 (1060).

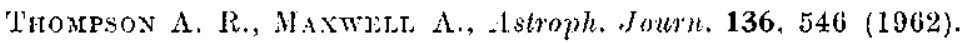

VAN DE HULST 11. C.. in The Sum, edit. Kuiper, 'The Vniversity Press, Chicago, 290-300 (1953).

Warwick C. S., Ihatritz M. W., Joum. Geoph. Res. 67, 1317 (1962).

Weiss A. A., Austral. Joum. Phys. 18, 167 (1965).

WeIss A. A., SHeridan K. V., Joum. Phys. Soc. Japan 17 Suppl. A-II, $223(1962)$.

Weiss A. A., S'Tewater R. 'T., Austral. Journ. Phys. 18, 143 (1965).

Westrold K. (., Austral. Journ. Sei. Res. A, 2, 169 (1949).

WiLd J. P., Austral. Joum. Sai. Ries. A, 3, 399 (1950).

WILD J. P., Journ. L'hys. Soo. Japan 17 Suppl. A.II, 249 (1962).

Wrid J. P., Murkar J. D., Rowe W. (., Austral. Journ. Sci. Res. A, 7, 439 (1954).

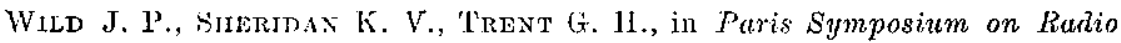
Astronomy (1959).

Winckler J. R., Journ. Geoph. Res. 65, 1331 (1960).

Winckler J. R., Bhavsar P. D., Journ. Geoph. Res. 65, 2637 (1960).

Winckler J. R., Bitavsar P. I)., Peterson L., Journ. Geoph. Res. 66, 995 (1961).

Winckler J. R., Masley A. J., MaY T.C., Journ. Geoph. Res. 66, 1023 (1961). 